\title{
Molecular and Ionized Gas Phases of an AGN-driven Outflow in a Typical Massive Galaxy at 22
}

Herrera-Camus, R.; Tacconi, L.; Genzel, R.; Schreiber, N. Foerster; Lutz, D.; Bolatto, A.; Wuyts, S.; Renzini, A.; Lilly, S.; Belli, S.

Total number of authors:

25

Published in:

Astrophysical Journal

Link to article, DOI:

10.3847/1538-4357/aaf6a7

Publication date:

2019

Document Version

Publisher's PDF, also known as Version of record

Link back to DTU Orbit

Citation (APA):

Herrera-Camus, R., Tacconi, L., Genzel, R., Schreiber, N. F., Lutz, D., Bolatto, A., Wuyts, S., Renzini, A., Lilly, S., Belli, S., Uebler, H., Shimizu, T., Davies, R., Sturm, E., Combes, F., Freundlich, J., Garcia-Burillo, S., Cox, P., Burkert, A., ... Weiss, A. (2019). Molecular and lonized Gas Phases of an AGN-driven Outflow in a Typical Massive Galaxy at z 2. Astrophysical Journal, 871(1). https://doi.org/10.3847/1538-4357/aaf6a7

\section{General rights}

Copyright and moral rights for the publications made accessible in the public portal are retained by the authors and/or other copyright owners and it is a condition of accessing publications that users recognise and abide by the legal requirements associated with these rights.

- Users may download and print one copy of any publication from the public portal for the purpose of private study or research.

- You may not further distribute the material or use it for any profit-making activity or commercial gain

- You may freely distribute the URL identifying the publication in the public portal 


\title{
Molecular and Ionized Gas Phases of an AGN-driven Outflow in a Typical Massive Galaxy at $z \approx 2$
}

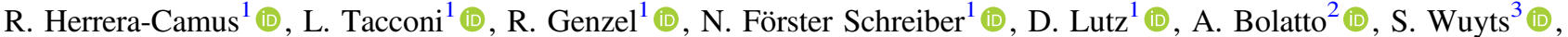 \\ A. Renzini ${ }^{4}$ (i) , S. Lilly ${ }^{5}$ (D), S. Belli ${ }^{1}$ (D), H. Übler ${ }^{1}$ (D), T. Shimizu ${ }^{1}$ (iD), R. Davies ${ }^{1}$, E. Sturm ${ }^{1}$ (i), F. Combes ${ }^{6}$ (D), J. Freundlich ${ }^{7}$,

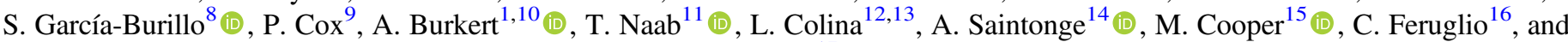 \\ A. Weiss ${ }^{17}$ (i) \\ ${ }^{1}$ Max-Planck-Institut für Extraterrestrische Physik (MPE), Giessenbachstr., D-85748 Garching, Germany; rhc@mpe.mpg.de \\ 2 Department of Astronomy, University of Maryland, College Park, MD 20742-2421, USA \\ ${ }^{3}$ Department of Physics, University of Bath, Claverton Down, Bath, BA2 7AY, UK \\ ${ }_{5}^{4}$ INAF-Osservatorio Astronomico di Padova, Vicolo dell'Osservatorio 5, I-35122 Padova, Italy \\ ${ }^{5}$ Department of Physics, Institute for Astronomy, ETH Zurich, CH-8093 Zurich, Switzerland \\ ${ }^{6}$ Observatoire de Paris, LERMA, College de France, CNRS, PSL Univ., Sorbonne Univ. UPMC, F-75014, Paris, France \\ ${ }^{7}$ Centre for Astrophysics and Planetary Science, Racah Institute of Physics, The Hebrew University, Jerusalem 91904, Israel \\ ${ }^{8}$ Observatorio Astronómico Nacional-OAN, Observatorio de Madrid, Alfonso XII, 3, E-28014-Madrid, Spain \\ ${ }^{9}$ CNRS, UMR 7095, Institut d'Astrophysique de Paris, F-75014 Paris, France \\ ${ }^{10}$ Universittäs-Sternwarte Ludwig-Maximilians-Universität (USM), Scheinerstr. 1, München, D-81679, Germany \\ ${ }^{11}$ Max-Planck Institute for Astrophysics, Karl Schwarzschildstrasse 1, D-85748 Garching, Germany \\ ${ }^{12}$ Centro de Astrobiología (CSIC/INTA), Ctra de Torrejón a Ajalvir, km 4, E-28850, Torrejón de Ardoz, Madrid, Spain \\ ${ }^{13}$ Cosmic Dawn Center (DAWN), Niels Bohr Institute, University of Copenhagen/DTU-Space, Technical University of Denmark, Denmark \\ ${ }^{14}$ Department of Physics \& Astronomy, University College London, Gower Place, London WC1E 6BT, UK \\ ${ }^{15}$ Department of Physics and Astronomy, Frederick Reines Hall, University of California, Irvine, CA 92697, USA \\ ${ }^{16}$ INAF Osservatorio Astronomico di Trieste, Via G.B. Tiepolo 11, I-34143 Trieste, Italy \\ ${ }^{17}$ Max-Planck-Institut für Radioastronomie (MPIfR), Auf dem Hügel 69, D-53121 Bonn, FRG, Germany \\ Received 2018 July 17; revised 2018 November 8; accepted 2018 December 2; published 2019 January 21
}

\begin{abstract}
Nuclear outflows driven by accreting massive black holes are one of the main feedback mechanisms invoked at high- $z$ to reproduce the distinct separation between star-forming disk galaxies and quiescent spheroidal systems. Yet our knowledge of feedback at high- $z$ remains limited by the lack of observations of the multiple gas phases in galaxy outflows. In this work, we use new deep, high spatial resolution ALMA CO(3-2) and archival Very Large Telescope/SINFONI H $\alpha$ observations to study the molecular and ionized components of the active galactic nucleus (AGN)-driven outflow in zC400528, a massive main-sequence galaxy at $z=2.3$ in the process of quenching. We detect a powerful molecular outflow that shows a positive velocity gradient before a turnover and extends for at least $\sim 10 \mathrm{kpc}$ from the nuclear region, about three times the projected size of the ionized wind. The molecular gas in the outflow does not reach velocities high enough to escape the galaxy and is therefore expected to be reaccreted. Keeping in mind the various assumptions involved in the analysis, we find that the mass and energetics of the outflow are dominated by the molecular phase. The AGN-driven outflow in zC400528 is powerful enough to deplete the molecular gas reservoir on a timescale comparable to that needed to exhaust it by star formation. This suggests that the nuclear outflow is one of the main quenching engines at work in the observed suppression of the central star formation activity in $\mathrm{zC} 400528$.
\end{abstract}

Key words: galaxies: active - galaxies: evolution - galaxies: high-redshift - galaxies: ISM

\section{Introduction}

Powerful galaxy outflows induced by active galactic nuclei (AGNs) have been invoked as one of the main drivers behind the transition experienced by massive star-forming galaxies during the main epoch of star formation activity into "red and dead" systems (e.g., Silk \& Rees 1998; Springel et al. 2005; King \& Pounds 2015). Outflows are expected to play (at least) a twofold role in quenching the star formation activity in massive galaxies. First, they have the power to expel the starforming material in nuclear regions to large distances ( $\gtrsim 5 \mathrm{kpc})$ on short timescales ( $\lesssim 1$ Gyr; ejective feedback; e.g., Feruglio et al. 2010; Sturm et al. 2011; Cicone et al. 2014; Morganti et al. 2016; Veilleux et al. 2017). Second, outflows can transfer enough radiative energy to the circumgalactic medium to drastically reduce the gas accretion rate onto the host galaxy (preventive feedback; e.g., Di Matteo et al. 2005; Gabor et al. 2011; van de Voort et al. 2011).
To quantify the impact of AGN feedback in galaxy evolution, it is important to understand the complex, multiphase nature of AGN-driven outflows. This is a challenging task that requires probing winds on a wide range of physical scales $(\sim 1 \mathrm{pc}-10 \mathrm{kpc})$, temperatures $\left(\sim 10^{2}-10^{7} \mathrm{~K}\right)$, and densities $\left(\sim 10^{2}-10^{8} \mathrm{~cm}^{-3}\right)$. The multiple outflow components include (1) very hot, quasi-relativistic winds from accretion disks observed on $\sim \mathrm{pc}$ scales in X-ray spectra (Nardini et al. 2015; Tombesi et al. 2015) and (2) hundred to kpc-scale atomic, molecular, and ionized winds traced by a variety of lines in absorption (e.g., H I, OH, Na I D, and Fe II; Rupke et al. 2002; Sturm et al. 2011; Kornei et al. 2012; González-Alfonso et al. 2017) and emission (e.g., $\mathrm{CO},[\mathrm{C} \mathrm{II}], \mathrm{H} \alpha$, [O III], etc.; Rupke \& Veilleux 2011; Contursi et al. 2013; García-Burillo et al. 2014; Harrison et al. 2016; Janssen et al. 2016; Woo et al. 2016; Concas et al. 2017). Accurate measurements of the extent, mass, and energetics of AGN-driven outflows can only be achieved when more than one gas-phase measurement is 
available, and even then, they are challenging (e.g., Mrk 231; Feruglio et al. 2010; Alatalo 2015; Morganti et al. 2016). Part of the problem is that the electron density and the CO-to- $\mathrm{H}_{2}$ factor are required to convert ionized and molecular luminosities into outflow masses, respectively. These quantities are poorly constrained and can introduce up to an order of magnitude uncertainty in the derived outflow masses.

Powerful AGN-driven outflows are found to be ubiquitous in typical massive galaxies at $z \sim 2$ (e.g., Förster Schreiber et al. 2014, 2018b; Genzel et al. 2014; Leung et al. 2017), the epoch when both star formation and nuclear activity peak. These outflows, mostly detected in ionized gas in near-infrared spectroscopic surveys, are fast $\left(v_{\text {out }} \sim 1000-2000 \mathrm{~km} \mathrm{~s}^{-1}\right)$, can extend to large scales $\left(R_{\text {out }} \sim 5-10 \mathrm{kpc}\right)$, and have massloading factors $\left(\eta \equiv \dot{M}_{\text {out }} / \mathrm{SFR}\right)$ in the $\eta \sim 0.1-2$ range (e.g., Genzel et al. 2014; Förster Schreiber et al. 2018b).

One of the main candidates to explain the observed suppression of star formation activity from nuclear regions outward in massive, main-sequence (MS) galaxies at $z \sim 2$ (also referred as inside-out quenching or growth; Tacchella et al. 2015a; Barro et al. 2016; Nelson et al. 2016) is AGN feedback in the form of outflows. However, this would probably require mass-loading factors higher than those measured in the ionized phase, which is the reason why it is so important to detect the molecular gas in outflows-most likely the wind phase carrying most of the ejected mass from the host galaxy (e.g., see compilation in Fiore et al. 2017).

Wideband receivers on new or upgraded millimeter-wave arrays such as the Atacama Large Millimeter/submillimeter Array (ALMA) and NOrthern Extended Millimeter Array (NOEMA) opened new windows to detect and characterize the faint (relative to the disk luminosity) molecular outflow signatures in quasi-stellar objects (QSOs; e.g., Vayner et al. 2017; Brusa et al. 2018; Fan et al. 2018) and typical massive galaxies between $z \sim 1$ and 3 . In this paper, we present one case where we combine sensitive, high spatial resolution ALMA and Very Large Telescope (VLT) observations to study the molecular and ionized gas phases of the powerful outflow detected in $\mathrm{zC} 400528$ at $z \sim 2$.

This paper is organized as follows. In Section 2, we summarize the properties of $\mathrm{zC} 400528$. In Section 3, we describe the observations and data reduction. In Section 4, we describe the properties (mass, size, energetics) of the molecular gas in the disk and outflow. In Section 5, we compare the molecular and ionized phases of the outflow. In Section 6, we discuss whether the ejected molecular gas can escape the host, the impact of AGN flickering and the expansion of the outflow, and the effect of AGN feedback in quenching the star formation activity in the galaxy. Finally, in Section 7, we present our conclusions.

\section{The Target of Study: $\mathrm{zC} 400528$}

The target of study, zC400528 (R.A. $=09: 59: 47.6$, decl. $=$ $+01: 44: 19.0)$, is a massive $\left(M_{\star}=1.0 \times 10^{11} M_{\odot} ;\right.$ Förster Schreiber et al. 2018a) star-forming galaxy at $z_{\mathrm{H} \alpha}=2.38$ (Förster Schreiber et al. 2014). The rate of star formation measured in this galaxy varies by a factor of $\sim 4$ depending on the tracer (for a detailed discussion see Tacchella et al. 2018). The star formation rate (SFR) value from evolutionary synthesis modeling of the optical to near-infrared spectral energy distribution is $\mathrm{SFR}_{\mathrm{SED}}=$ $148 M_{\odot} \mathrm{yr}^{-1}$. The same value of SFR is obtained using the UV continuum emission corrected for attenuation based on the UV continuum slope $\left(\mathrm{SFR}_{\mathrm{UV} \text {, corr }}=148 M_{\odot} \mathrm{yr}^{-1}\right)$. A more direct

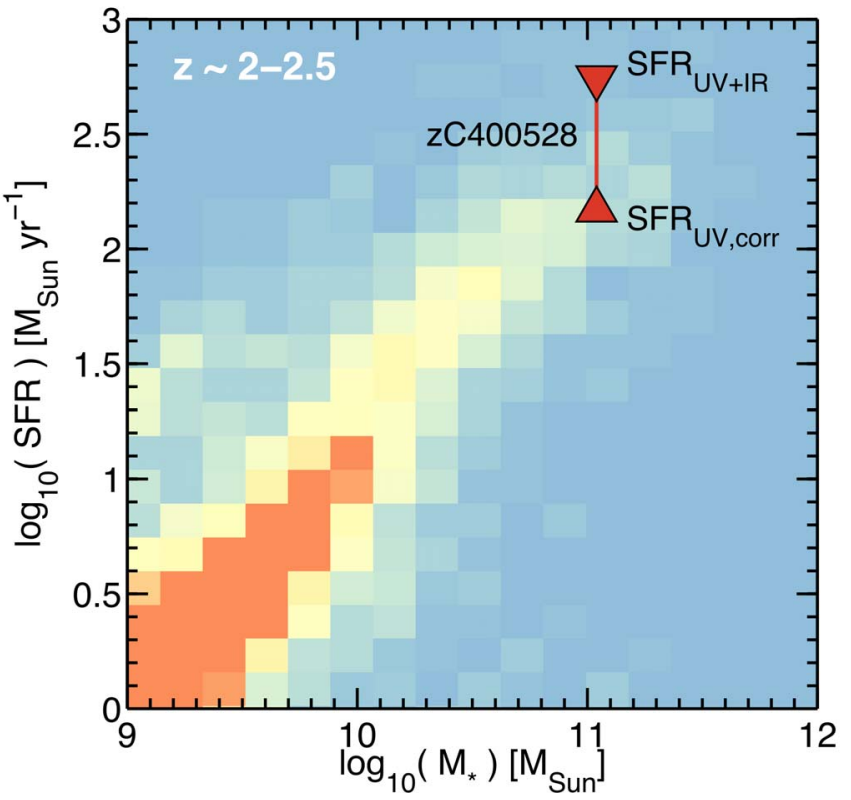

Figure 1. Location of $\mathrm{zC} 400528$ (red triangles) in the stellar mass-star formation rate plane for galaxies in the redshift range $2 \leqslant z \leqslant 2.5$ selected from 3D HST (Skelton et al. 2014). With a stellar mass of $M_{\star}=1.1 \times$ $10^{11} M_{\odot}$ and an SFR of 148 (from SED fitting or UV emission corrected by dust attenuation) or 556 (from the combination of UV and $100 \mu \mathrm{m}$ dust emission) $M_{\odot} \mathrm{yr}^{-1}, \mathrm{zC} 400528$ lies at the massive end of the MS of starforming galaxies.

determination of the dust content of $\mathrm{zC} 400528$, although at the cost of spatial resolution, comes from a $\lambda_{\text {obs }}=100 \mu \mathrm{m}$ (restframe $\left.\lambda_{\text {rest }}=29.5 \mu \mathrm{m}\right)$ dust continuum detection with the Herschel PACS (Pilbratt et al. 2010; Poglitsch et al. 2010). The combined UV and $100 \mu \mathrm{m}$ star formation rate is $\mathrm{SFR}_{\mathrm{UV}+\mathrm{IR}}=$ $556 M_{\odot} \mathrm{yr}^{-1}$, a factor of 3.7 times higher than $\mathrm{SFR}_{\mathrm{UV} \text {,corr }}$. Note, however, that this composite SFR needs to be interpreted with caution. The global continuum dust emission at rest frame $29 \mu \mathrm{m}$ is most likely contaminated by emission from dust heated by supermassive black hole activity ( 20\%-60\%; e.g., Brown et al. 2018) and evolved stars ( 20\%-50\%; e.g., Leroy et al. 2012; Herrera-Camus et al. 2015; Narayanan et al. 2015). Finally, zC400528 is detected in the COSMOS VLA radio data (Schinnerer et al. 2010) and has a flux at observed $1.4 \mathrm{GHz}$ of $67 \mu \mathrm{mJy}$, which can be converted into a star formation rate of $\mathrm{SFR}_{1.4 \mathrm{GHz}} \approx 570 M_{\odot} \mathrm{yr}^{-1}$ following the calibration by Murphy et al. (2011). This value is comparable to $S_{F R}+I R$, but it is important to keep in mind that the radio continuum emission in radio-quiet AGNs has a contribution from the accretion disk corona (e.g., Smith et al. 2016; Wong et al. 2016). In practice, the true SFR of zC400528 most likely lies between $\mathrm{SFR}_{\mathrm{UV} \text {, corr }}$ (or $\mathrm{SFR}_{\mathrm{SED}}$ ) and $\mathrm{SFR}_{\mathrm{UV}+\mathrm{IR}}$ (or $\mathrm{SFR}_{1.4 \mathrm{GHz}}$ ). All of these star formation tracers have age sensitivities between 0 and $100 \mathrm{Myr}$, with mean ages for the stellar populations contributing to the FUV and rest-frame $24 \mu \mathrm{m}$ emission of 10 and $5 \mathrm{Myr}$, respectively (Kennicutt \& Evans 2012).

Figure 1 shows the position of $\mathrm{zC} 400528$ in the $M_{\star}-\mathrm{SFR}$ plane of galaxies at $z \sim 2-2.5$. According to the parameterization of Whitaker et al. (2014), zC400528 lies on top of or a factor of $\sim 4$ above the "MS" of star-forming galaxies, depending on whether we assume $\mathrm{SFR}_{\mathrm{UV} \text {,corr }}$ or $\mathrm{SFR}_{\mathrm{UV}+\mathrm{IR}}$, respectively. 
As discussed in detail in Förster Schreiber et al. (2014), zC400528 shows evidence for AGN activity. First, the high $[\mathrm{N} \mathrm{II}] / \mathrm{H} \alpha$ peak line ratio $(\sim 0.8)$ and fast ionized outflow $\left(\sim 1000 \mathrm{~km} \mathrm{~s}^{-1}\right)$ observed in zC400528 (Genzel et al. 2014) are only comparable to those found in AGN galaxies at $z \sim 2$. Massive galaxies with star formation-driven winds at similar redshift have typical $[\mathrm{N} \mathrm{II}] / \mathrm{H} \alpha$ line ratios and wind speeds a factor of 2 lower (Förster Schreiber et al. 2018a).

Second, zC400528 satisfies the AGN criteria in the $\log \left(S_{5.8} / S_{3.6}\right)$ versus $\log \left(S_{8.0} / S_{4.5}\right)$ diagram of Lacy et al. (2004) and lies near the boundary of the $\log \left(S_{24} / S_{100}\right)$ versus $\log \left(S_{8.0} / S_{3.6}\right)$ diagram of Kirkpatrick et al. (2013). Unfortunately, the galaxy remains undetected in the Chandra X-ray data of the COSMOS field (Elvis et al. 2009). In the Appendix, we discuss how we measured the AGN luminosity of $\mathrm{zC} 400528$ using the relation between the rest-frame $\sim 7 \mu \mathrm{m}$ dust continuum and X-ray luminosity (e.g., Lutz et al. 2004; Mateos et al. 2015).

Also, in the study of Tacchella et al. (2015a), zC400528 is one of the galaxies that shows evidence for inside-out quenching on short timescales $(\lesssim 1 \mathrm{Gyr})$ in the inner $\sim \mathrm{kpc}$ region (see their Figure S4, last panel). From a statistical point of view, we also expect that zC400528 will experience star formation quenching as the fraction of quiescent galaxies with the stellar mass of $\mathrm{zC} 400528$ increases from $\sim 20 \%$ at $2<z<2.5$ to $\sim 70 \%$ at $1<z<1.5$ (Muzzin et al. 2013).

Throughout this paper, we adopt a redshift for $\mathrm{zC} 400528$ of $z=2.387$ based on the detections of the $\mathrm{H} \alpha$ (Förster Schreiber et al. 2014) and $\mathrm{CO}(3-2)$ lines (this work). This redshift value sets the systemic velocity for all of the kinematic features discussed in the analysis. Assuming a cosmology with $H_{0}=70 \mathrm{~km} \mathrm{~s}^{-1} \mathrm{Mpc}^{-1}, \Omega_{M}=0.27$, and $\Omega_{\Lambda}=0.73$, at $z=$ $2.38,1^{\prime \prime}$ corresponds to a linear scale of $8.3 \mathrm{kpc}$.

\section{Observations and Data Reduction}

The main goal of our observations was to detect and spatially resolve the molecular gas outflow in $\mathrm{zC} 400528$ using the redshifted $\mathrm{CO}(3-2)$ line emission as a tracer of molecular gas. Our target was first observed by ALMA in Cycle 2 for a total of $0.75 \mathrm{hr}(0.6 \mathrm{hr}$ on-source $)$ as part of project 2013.1.00092.S (PI: R. Genzel). The observations were carried out in the C34$5 / 6$ configuration, resulting in an angular resolution of 1 !" $1 \times 0 . " 6$ in Band 3. During the next ALMA cycle, our source was observed as part of project 2015.1.00220.S (PI: R. Genzel) in a more extended configuration, C40-6, resulting in an angular resolution of 0 ." $4 \times 0$ ". 3 in Band 3. The total observation time was $5.4 \mathrm{hr}$ ( $4.5 \mathrm{hr}$ on-source). In both cases, the spectral setup included one of the spectral windows in Band 3 centered around the redshifted $\mathrm{CO}(3-2)$ line emission $\left(\nu_{\mathrm{obs}}=102.004 \mathrm{GHz}\right)$.

The data were processed using the Common Astronomy Software Applications package (CASA; McMullin et al. 2007). The pipeline-calibrated interferometric visibilities delivered by ALMA from both cycles were then combined and imaged at $50 \mathrm{~km} \mathrm{~s}^{-1}$ resolution using natural weighting. In the combination process, we took into account the different CASA visibility weights applied during Cycles 2 and 3 using the CASA task STATWT. ${ }^{18}$ The size of the restoring beam of the combined data was 0 " $51 \times 0$ ". $47(4.2 \times 3.9 \mathrm{kpc})$ at a position angle of

\footnotetext{
$\overline{18}$ For more details, see https://casaguides.nrao.edu/index.php/DataWeights AndCombination.
}

$\mathrm{PA}=-12^{\circ} .13$. By combining the data from the more extended and compact array configurations, we achieved high enough spatial resolution to resolve the galaxy and, at the same time, improve our ability to detect and characterize a potential extended, more diffuse component of the molecular outflow.

All values were primary beam-corrected for all quantitative analyses. We reached an rms noise of $0.08 \mathrm{mJy}^{-1}$ beam $^{-1}$ in $50 \mathrm{~km} \mathrm{~s}^{-1}$ channels for $\mathrm{CO}(3-2)$. No continuum emission was detected at $100 \mathrm{GHz}$.

For a detailed description of the observations of the $\mathrm{H} \alpha$ and [N II] lines in $\mathrm{zC} 400528$ carried out with the near-IR integral field spectrograph SINFONI (Eisenhauer et al. 2003) at the VLT, we refer to Förster Schreiber et al. (2014). In summary, the galaxy was observed in the $K$ band for an on-source time of $4 \mathrm{hr}$ using a natural guide star for the adaptive optics correction. The achieved angular resolution (PSF FWHM) of the final reduced data was 0 " 15 .

\section{Results \\ 4.1. The Molecular Disk}

Figure 2 shows the $\mathrm{CO}(3-2)$ integrated intensity and velocity maps of zC400528. We measure an integrated $\mathrm{CO}(3-2)$ flux of $F_{\mathrm{CO}}=1.11 \pm 0.05 \mathrm{Jy} \mathrm{km} \mathrm{s}^{-1}$, which corresponds to a luminosity of $L_{\mathrm{CO}}=3.45 \times 10^{10} L_{\odot} \cdot{ }^{19}$ The molecular gas mass $\left(M_{\text {mol,disk }}\right)$, assuming a conversion factor $\alpha_{\mathrm{CO}(1-0), \mathrm{T} 18}=$ $4.3 M_{\odot}\left(\mathrm{K} \mathrm{km} \mathrm{s}^{-1} \mathrm{pc}^{-2}\right)^{-1}$ (Tacconi et al. 2018) ${ }^{20}$ and a velocity-integrated Rayleigh-Jeans brightness temperature line ratio $R_{13}=1.3$ (e.g., Dannerbauer et al. 2009; Bolatto et al. 2015; Daddi et al. 2015), is $M_{\text {mol,disk }}=1.1 \times 10^{11} M_{\odot} \cdot{ }^{21}$ This corresponds to a gas-to-stellar ratio of $\mu_{\mathrm{mol}, \text { disk }}=M_{\mathrm{mol}} / M_{*} \approx$ 1 , which is consistent with the typical gas-mass fractions observed in massive star-forming galaxies at $z \sim 2$ (e.g., Tacconi et al. 2010, 2013, 2018).

If we add up the stellar and molecular gas masses, we obtain $M_{*}+M_{\text {mol,disk }} \approx 2 \times 10^{11} M_{\odot}$, which is comparable within the uncertainties to the dynamical mass derived from the ionized gas kinematics, i.e., $M_{\mathrm{dyn}}=1.3_{-0.6}^{+1.5} \times 10^{11} M_{\odot}$ (Förster Schreiber et al. 2018a).

The integrated velocity map in the right panel of Figure 2 shows that the molecular disk is rotating approximately in the east-west direction, consistent with the observed rotation in the ionized gas (Newman et al. 2013; Förster Schreiber et al. 2018a).

Figure 3 (left) compares the spatial distribution of the molecular gas, the ionized gas disk traced by $\mathrm{H} \alpha$ emission (Förster Schreiber et al. 2014), and the stellar mass traced by the Hubble Space Telescope (HST) $H$ band (Tacchella et al. 2015b). Both molecular and ionized gas components share a similar spatial structure, including a more diffuse component that extends toward the southeast region. The peak of the $H$-band emission is shifted $\sim 0$ !" 2 northeast with respect to the

\footnotetext{
19 This measurement does not include the contribution of the outflow to the $\mathrm{CO}(3-2)$ emission. Please see Section 4.2.1 for a description of how the molecular gas emission of the disk and outflow was disentangled.

${ }^{20}$ The $\alpha_{\mathrm{CO}}$ conversion function in Tacconi et al. (2018) corresponds to the geometric mean between the $\alpha_{\mathrm{CO}}$ recipes as a function of metallicity of Bolatto et al. (2013b) and Genzel et al. (2015). zC400528 is a massive $\left(M_{*}=1.1 \times 10^{11} M_{\odot}\right)$ MS galaxy at $z=2.38$ that according to the scaling relations in Tacconi et al. (2018), has a metallicity near solar $(12+\log (\mathrm{O} / \mathrm{H})=8.6)$ on the Pettini \& Pagel (2004) scale.

21 The calculation of the molecular gas mass includes a helium correction factor of 1.36 .
} 

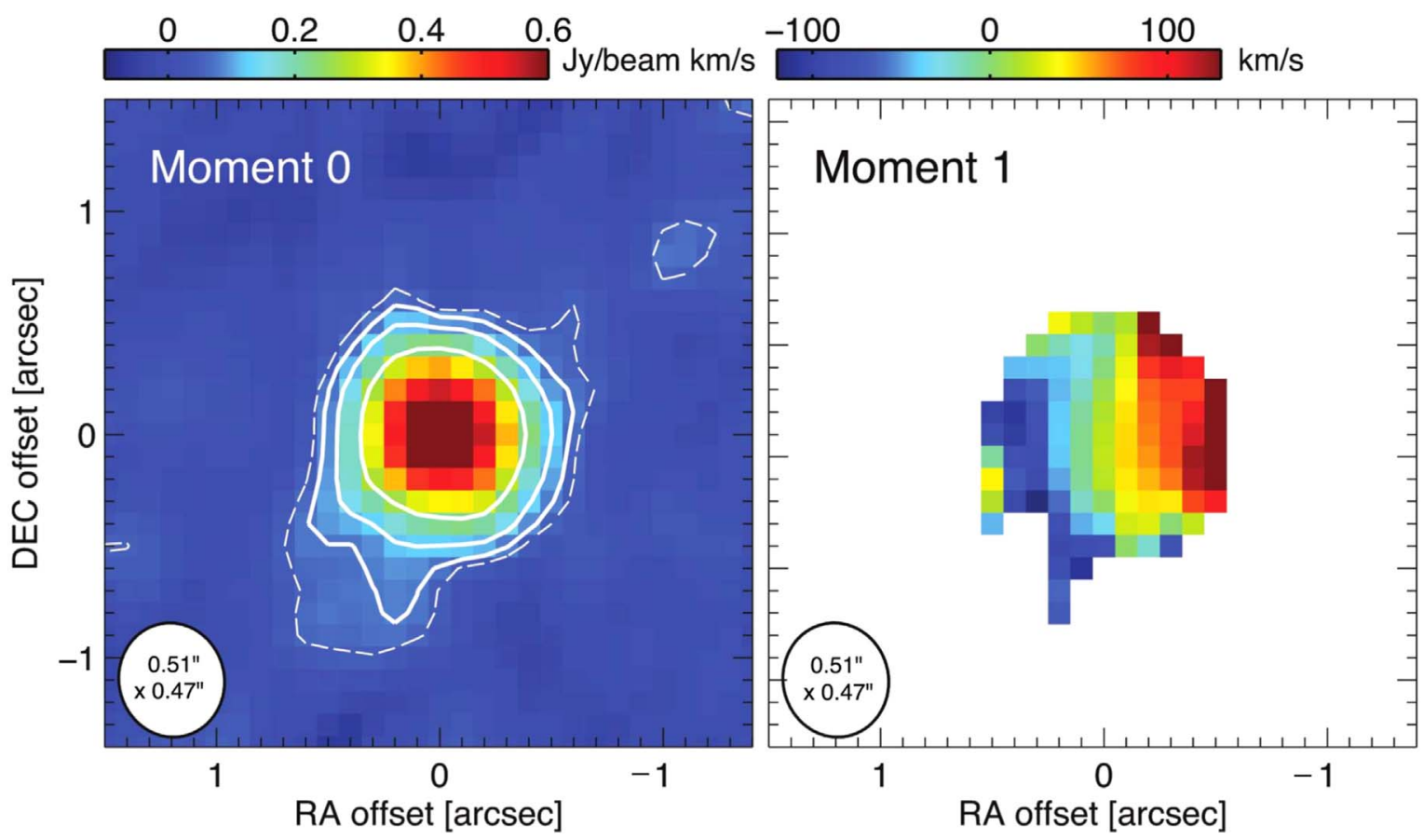

Figure 2. ALMA CO(3-2) integrated intensity (left) and velocity (right) maps of zC400528. The contours in the left panel correspond to $2 \sigma$ (dashed), $3 \sigma, 5 \sigma$, and $10 \sigma$. For the velocity map, we only include regions with integrated flux emission higher than $3 \sigma$. The synthesized beam is illustrated in the bottom left corner. The color bar at the top of each panel indicates the intensity scale.

peak of the ALMA $\mathrm{CO}(3-2)$ emission. The astrometric precision of our ALMA data is $\sim 0$ !" $025,{ }^{22}$ which is at least a factor of $\sim 10$ better than the astrometrical accuracy of the HST data. Therefore, it is not possible to determine whether the spatial offset is due to physical reasons or astrometric errors.

The right panel of Figure 3 shows the $\mathrm{CO}(3-2)$ and $\mathrm{H} \alpha$ $+[\mathrm{N} \mathrm{II}]$ spectrum from within a 0 " 6 radius circular aperture centered at the peak of emission (the systemic velocity corresponds to a redshift of $z=2.387$; see Section 2). The $[\mathrm{N} \mathrm{II}] / \mathrm{H} \alpha$ line peak ratio is 0.8 (see also Genzel et al. 2014), which is characteristic of galaxies where the AGN contributes to the ionizing radiation or shocks affect the ionization balance (e.g., Kewley et al. 2013; Newman et al. 2014). As described in detail in Förster Schreiber et al. (2014) and Genzel et al. (2014), an ionized outflow is detected as a strong nuclear broad component in the $\mathrm{H} \alpha$ spectrum (detected out to velocities $\sim \pm 1000 \mathrm{~km} \mathrm{~s}^{-1}$ relative to the systemic velocity). The broad $\mathrm{H} \alpha$ emission is resolved with a spatial resolution of 0 !" 15 $(\sim 1.2 \mathrm{kpc})$. In the case of the $\mathrm{CO}(3-2)$ spectrum, we observe a high-velocity wing on the red side $\left([+300,+500] \mathrm{km} \mathrm{s}^{-1}\right)$ that is likely the molecular component of the outflow. We analyze and interpret this high-velocity feature in the $\mathrm{CO}(3-2)$ spectrum in the next section.

\subsection{The Molecular Outflow}

As a first step to study the high-velocity components in the ionized and molecular spectrum of zC400528, we make position-velocity $(\mathrm{P}-\mathrm{V})$ diagrams extracted along the vertical (south-north), horizontal (west-east), and $-45^{\circ}$ (southeastnorthwest) directions through the emission peak. The top panel in Figure 4 shows the results for the $\mathrm{CO}(3-2)$ data. We identify the rotation pattern of the galaxy in the $[-250,+250] \mathrm{km} \mathrm{s}^{-1}$

\footnotetext{
22 According to the ALMA Technical Handbook, Chapter 10.6.6, Astrometric Observations.
}

velocity range and a high-velocity $\left(v \gtrsim+300 \mathrm{~km} \mathrm{~s}^{-1}\right)$ component along all of the examined directions that preferentially extends along the northwest direction for about $\sim 1^{\prime \prime}$ (which corresponds to a projected distance of $\approx 8.4 \mathrm{kpc}$ ). The contours in the bottom panels of Figure 4 show the $\mathrm{P}-\mathrm{V}$ curves for the $\mathrm{H} \alpha$ and [N II] lines (the systemic velocity is set to match the $\mathrm{H} \alpha$ line central velocity) plotted on top of the $\mathrm{CO}(3-2) \mathrm{P}-\mathrm{V}$ diagram. On the receding side, the outflow is detected in both ionized and molecular gas, with the latter showing a more extended spatial distribution. On the other hand, there is no molecular counterpart to the high-velocity ionized gas observed at velocities $v \lesssim-300 \mathrm{~km} \mathrm{~s}^{-1}$.

\subsubsection{Disentangling the Molecular Emission from Disk and Outflow}

Quantifying the total amount of molecular gas in the outflow requires identifying and removing the $\mathrm{CO}(3-2)$ line emission associated with the rotating disk. To achieve this, here we use two different methods.

(1) Two-component Gaussian fit to the spectrum. The most common approach to disentangling emission from the disk and outflow in galaxies is to simultaneously fit two Gaussian profiles to the spectrum. The first Gaussian component centered at the systemic velocity represents the rotating disk, while the second component characterizes the outflow, with the caveat that it might include line emission at low velocities that is associated with the disk. We applied this method to the $\mathrm{CO}(3-2)$ spectrum extracted within a 0 ". 5 radius circular aperture centered at the peak of $\mathrm{CO}$ emission. The resulting two-Gaussian fit is shown in Figure 5 (bottom left panel). The component centered at the systemic velocity $\left(v_{0, \text { disk }}=-15 \mathrm{~km} \mathrm{~s}^{-1}\right)$ reaches a peak flux density of $S_{\text {peak, disk }}^{\mathrm{CO}}=2.4 \mathrm{mJy}$ and has a velocity width of $\mathrm{FWHM}_{\text {disk }}=277.3 \mathrm{~km} \mathrm{~s}^{-1}$. The second component, which we associate with the outflow, 

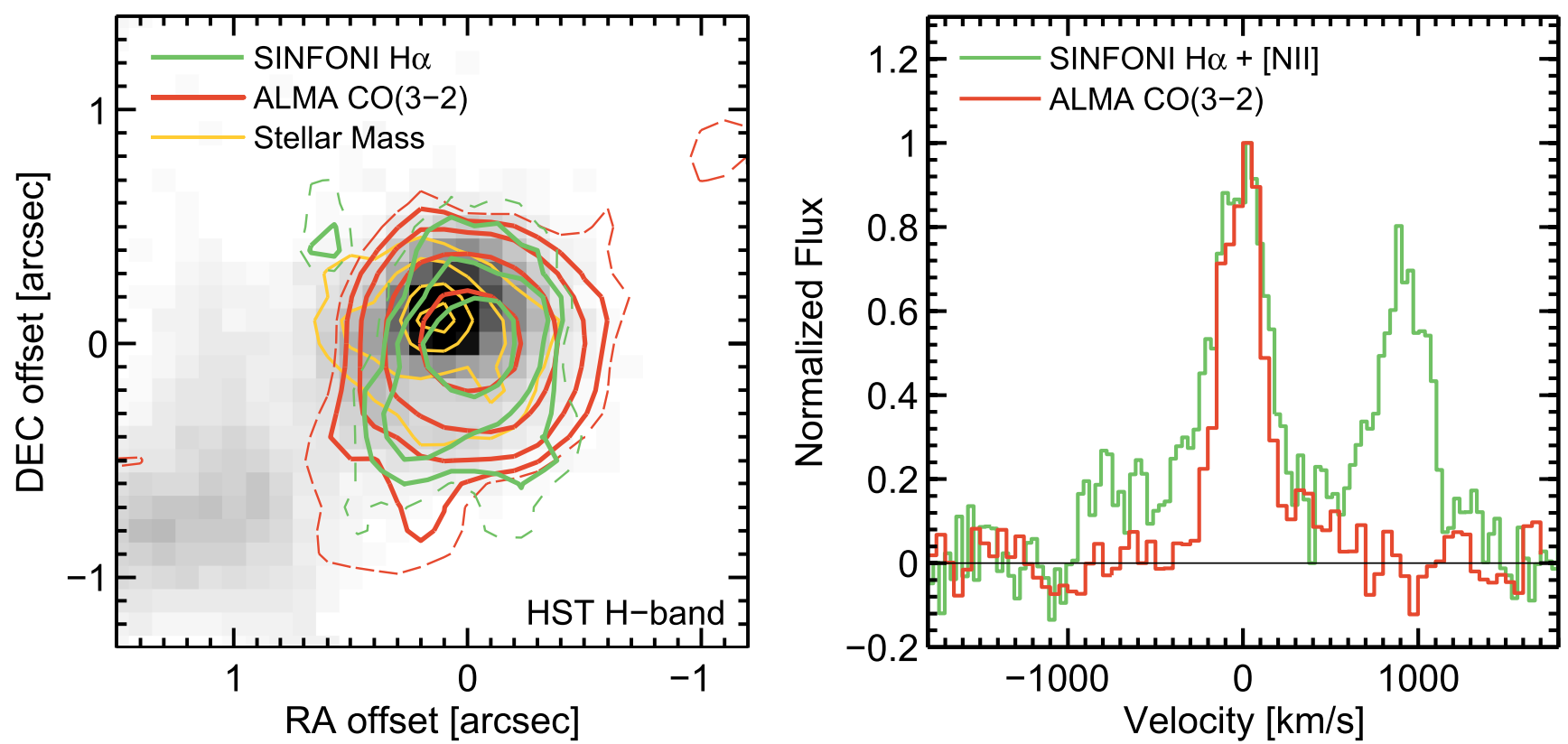

Figure 3. (Left) Contours from ALMA CO(3-2) (red) and SINFONI H $\alpha$ (green) integrated intensity maps and the stellar mass distribution (yellow) overlaid on a gray-scale $H S T H$-band image of zC400528. For the $\mathrm{CO}(3-2)$ and $\mathrm{H} \alpha$ data, the contours correspond to $2 \sigma$ (dashed), $3 \sigma, 5 \sigma, 10 \sigma$, and $20 \sigma$. The contours in stellar mass are $1.5,3,6$, and $9 \times 10^{8} M_{\odot}$. We observe that the $\mathrm{CO}(3-2)$ emission is offset $\sim 0 . \prime 2$ relative to the spatial position of the HST H-band and stellar mass maps. (Right) ALMA CO(3-2) and SINFONI $\mathrm{H} \alpha+[\mathrm{N}$ II] spectrum within a 0." 6 radius circular aperture centered at the peak of emission. The systemic velocity corresponds to a redshift of $z=2.38$. For both ionized and molecular tracers, there is evidence for a broad emission component associated with an AGN-driven outflow.

has a central velocity of $v_{0, \text { out }}=415 \mathrm{~km} \mathrm{~s}^{-1}$, a peak flux density of $S_{\text {peak,out }}^{\mathrm{CO}}=0.38 \mathrm{mJy}\left(\sim 0.16 \times S_{\text {peak,disk }}^{\mathrm{CO}}\right)$, and a velocity width $\mathrm{FWHM}_{\text {out }}=254.3 \mathrm{~km} \mathrm{~s}^{-1}$. The integrated $\mathrm{CO}(3-2)$ flux in the outflow component is $F_{\mathrm{CO}, \text { out }}=0.10 \mathrm{Jy} \mathrm{km} \mathrm{s}^{-1}$.

(2) Subtraction of a smooth model of the disk. Given that we have sufficient spatial resolution and signal-to-noise in our ALMA CO(3-2) data, here we try a different strategy to attempt to remove the disk material. The method consists of creating and subtracting from the original cube a smooth model of the rotating disk. This approach is similar to that used by Veilleux et al. (2017) to disentangle the $\mathrm{CO}(1-0)$ line emission associated with the molecular outflow in a luminous infrared galaxy.

We use the CASA task IMFIT to fit in each $50 \mathrm{~km} \mathrm{~s}^{-1}$ channel of the $\mathrm{CO}(3-2)$ cube a two-dimensional Gaussian to the image in the region where the source is detected. We then use the resulting fits in the $[-250,+250] \mathrm{km} \mathrm{s}^{-1}$ range to make a source model where the Gaussian position, orientation, and intensity changes as a function of velocity. The velocity range was chosen to encompass the bulk of the $\mathrm{CO}(3-2)$ line emission associated with the rotating disk as suggested by the two-component Gaussian fit, the observed line width of the $\mathrm{H} \alpha$ line $\left(\Delta v / 2 \approx 150 \mathrm{~km} \mathrm{~s}^{-1}\right.$; Förster Schreiber et al. 2018a), and the P-V diagram (Figure 4). In the last step, we removed the source model from each $50 \mathrm{~km} \mathrm{~s}^{-1}$ slice, resulting in a residual cube where the outflowing gas is now disentangled from the emission associated with the rotating disk.

The top panels in Figure 5 show the integrated intensity map calculated on the $[-250,+250] \mathrm{km} \mathrm{s}^{-1}$ range of the original cube (left) and the cube resulting after we subtract the rotating disk model (i.e., the residual cube; right). The model does a good job of removing the $\mathrm{CO}(3-2)$ line emission associated with the rotating disk, and we only observe a relatively high residual at the level of $\sim 0.06 \mathrm{Jy} \mathrm{km} \mathrm{s}^{-1}$ in the more diffuse and extended southeast component. The bottom left panel in Figure 5 shows the $\mathrm{CO}(3-2)$ spectrum integrated over a circular aperture with a radius of 0.15 . The black and green lines correspond to the spectrum extracted in the original cube and the residual cube, respectively. The integrated signal of the high-velocity red-wing emission in the residual cube is clear, and there is no evidence for a molecular outflow component on the approaching side of the spectrum. The bottom right panel of Figure 5 shows the integrated intensity map of the residual cube in the velocity range where we identified the red-wing emission $\left([+300,+700] \mathrm{km} \mathrm{s}^{-1}\right)$. The outflow emission peaks around 0 ". $2(\sim 2 \mathrm{kpc})$ west of the molecular disk center, and there is a tail of outflowing gas line emission that extends beyond the molecular disk toward the northwest for about $1^{\prime \prime}$, which corresponds to a projected distance of $\sim 8 \mathrm{kpc}$.

\subsubsection{The Structure of the Molecular Outflow}

A more detailed view of the molecular outflow components as a function of velocity is shown in the velocity channel map of the residual cube in Figure 6. For reference, overplotted on each panel are the contours from the velocity-integrated $\mathrm{CO}(3-2)$ emission of the rotating disk. The first significant outflow structure appears at $v=+300 \mathrm{~km} \mathrm{~s}^{-1}$ and is spatially coincident with the central region of the molecular gas disk. Between $v=+350$ and $+400 \mathrm{~km} \mathrm{~s}^{-1}$, the main component of the outflow moves toward the west about 0 !' 2 , which corresponds to a projected distance of $\sim 2 \mathrm{kpc}$. At $v=$ $+450 \mathrm{~km} \mathrm{~s}^{-1}$, the main outflow component starts to extend toward the north and continues until $v=+500 \mathrm{~km} \mathrm{~s}^{-1}$, which is the last channel at which we detect $\mathrm{CO}(3-2)$ line emission.

The last two panels in Figure 6 show the integrated intensity and velocity maps of the molecular outflow detected in the $[+250,+500] \mathrm{km} \mathrm{s}^{-1}$ range. The bulk of the line emission 


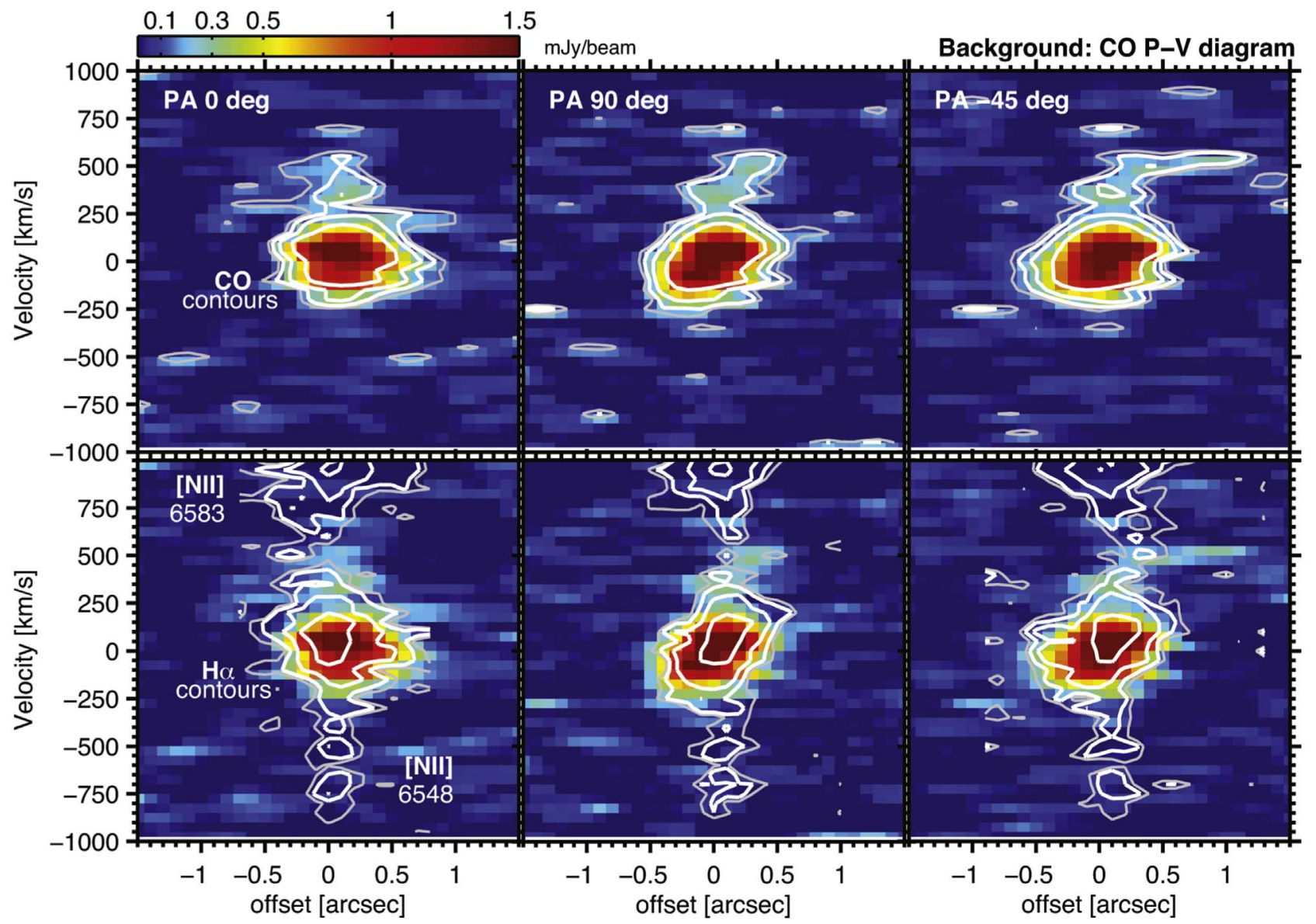

Figure 4. (Top panels) P-V diagram of zC400528 for the ALMA CO(3-2) data (background and contours) taken at a position angle of $0^{\circ}$ (left), $90^{\circ}$ (middle), and $-45^{\circ}$ (right) through the $\mathrm{CO}(3-2)$ emission peak. The contours show the $2 \sigma$ (gray), $3 \sigma, 6 \sigma$, and $10 \sigma$ (white) levels of emission. (Bottom panels) Similar to the top panels, but this time the contours are based on the $\mathrm{P}-\mathrm{V}$ diagram for the SINFONI $\mathrm{H} \alpha+\left[\mathrm{N}\right.$ II] data. High-velocity gas at $v \gtrsim+250 \mathrm{~km} \mathrm{~s}^{-1}$ is seen in both molecular and ionized emission.

associated with the outflow is shifted about $0 . " 2$ (projected distance of $\sim 2 \mathrm{kpc}$ ) west of the center of the molecular disk and extends to the north for approximately 0 ." 7 (projected distance of $\sim 6 \mathrm{kpc}$ ), where it shifts to the west in what seems to be a second outflow component-still connected to the main component-at about 1.'2 (projected distance of $\sim 10 \mathrm{kpc}$ ) from the center. One could suspect that this spatially extended feature of the outflow is in reality a tidal tail product of the interaction with a lower-mass companion, but visual inspection of a deep HST F160W image of the field reveals no stellar emission associated with a close neighbor in the northeast quadrant.

We observe a velocity gradient in the outflow: the gas velocity increases from $\sim+300$ to $\sim+500 \mathrm{~km} \mathrm{~s}^{-1}$ as the outflow extends north of the nucleus. This could be indicative of an accelerating continuous outflow. Another possibility could be that the ejection of molecular gas triggered during the last outflow burst had a distribution of velocities, so the faster ejecta components traveled further away than the slower components. The most distant outflow component in the northwest has a velocity of $\sim+350 \mathrm{~km} \mathrm{~s}^{-1}$, a lower velocity that could be the result of gravitational pull.

In summary, perhaps the most striking characteristic of the molecular outflow in zC400528 is its asymmetry: we only detect the receding component of the wind. In the local universe, it is not uncommon to observe asymmetric outflows.
For example, Pereira-Santaella et al. (2018) found that in four out of five spatially resolved molecular gas outflows in ultraluminous infrared galaxies (ULIRGs), the receding component of the wind is stronger than the approaching one. This is also true for the molecular outflow in Mrk 231 (Cicone et al. 2012; Feruglio et al. 2015). High-resolution simulations of $z \sim 2$ isolated disks also find that AGN-driven outflows are typically unipolar as a result of dense cloud structures in the vicinity of the black hole blocking the expansion in one direction (Gabor \& Bournaud 2014). Another possibility is that the molecular outflow on the approaching side experienced a change of phase, similar to what is observed in M82, where the dominant phase of the wind transitions from molecular to atomic at about $1 \mathrm{kpc}$ from the disk (Leroy et al. 2015).

\subsubsection{The Molecular Gas Mass in the Outflow}

For each outflow component, we measure $\mathrm{CO}(3-2)$ flux densities with Gaussian fits to the spectra in the residual cube extracted from the circular and elliptical apertures shown in Figure 7 . The flux densities and velocity dispersions $\left(\sigma_{\mathrm{CO}}\right)$ are listed in Table 1.

The velocity dispersions derived from the Gaussian fit to the spatially resolved outflow components 1,2 , and 3 are in the $\sigma_{\mathrm{CO}} \sim$ $60-170 \mathrm{~km} \mathrm{~s}^{-1}$ range. These are comparable to the velocity 


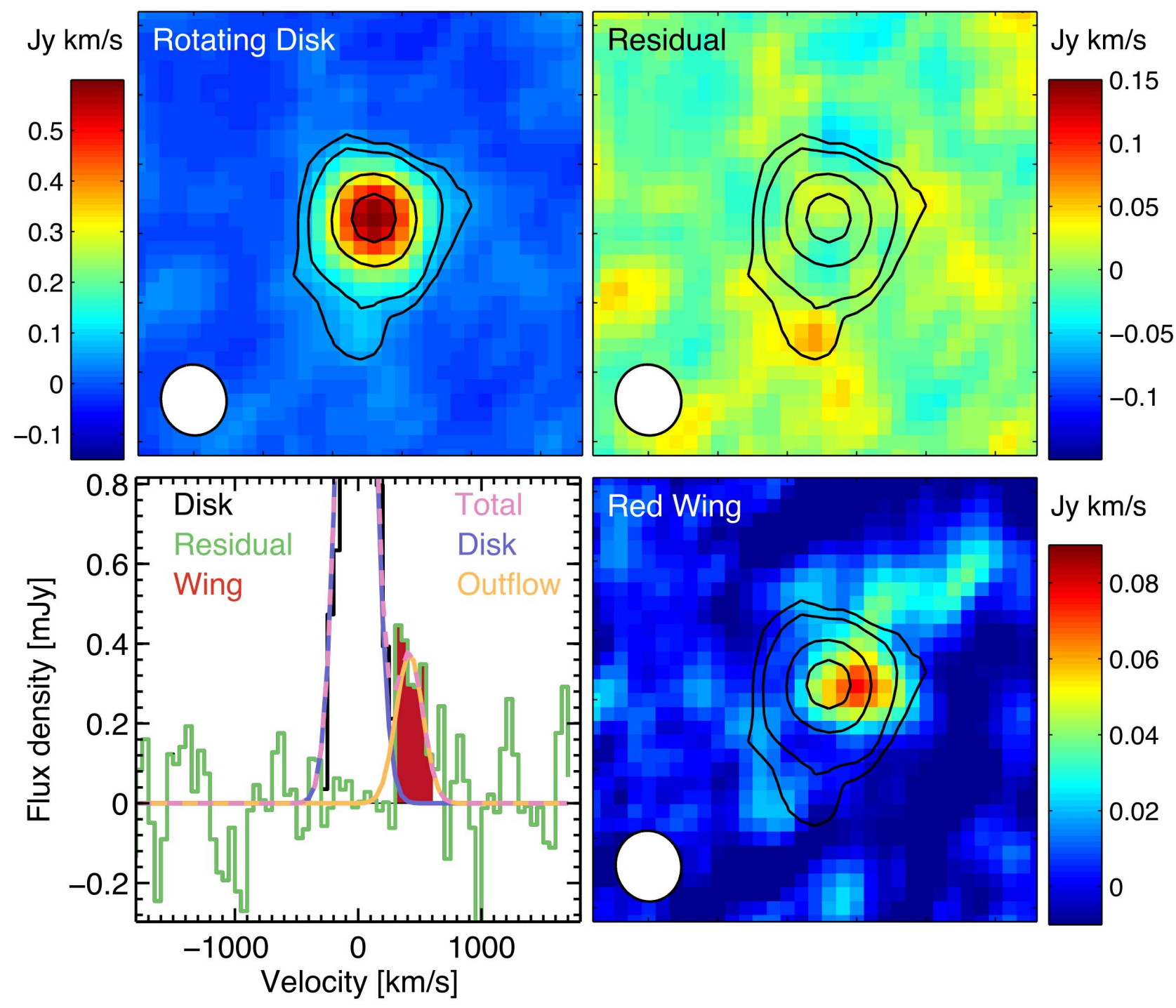

Figure 5. Analysis of the kinematic components of zC400528 based on a two-dimensional Gaussian fit to the $\mathrm{CO}(3-2)$ emission in the $50 \mathrm{~km} \mathrm{~s}{ }^{-1}$ channels. (Top panels) Integrated intensity maps in the $[-250,250] \mathrm{km} \mathrm{s}^{-1}$ range for the rotating disk component (left) and the residual component after subtracting the rotating disk (right). The synthesized beam is illustrated in the bottom left corner $\left(00^{\prime \prime} 51 \times 00^{\prime \prime} 47\right)$. The color bar indicates the intensity scale. (Bottom left panel) CO(3-2) spectrum of the rotating (disk) and residual (green) components within a 0 " 5 radius circular aperture centered 0.2 west of the peak of CO emission in the rotating disk. We also show the best two-component Gaussian fit to the $\mathrm{CO}(3-2)$ spectrum. (The disk and outflow components are shown in purple and yellow, respectively. The sum of the two components is shown in pink). In red, we show what we identify as the red-wing outflow material. (Bottom right panel) Intensity map of the red-wing component integrated between +300 and $+650 \mathrm{~km} \mathrm{~s}^{-1}$. The contours in each map represent the velocity-integrated $\mathrm{CO}(3-2)$ emission of the rotating disk. The separation between tick marks is $1^{\prime \prime}$.

dispersion observed in the spatially resolved molecular outflows of M82 $\left(\sigma_{\mathrm{CO}} \sim 30-70 \mathrm{~km} \mathrm{~s}^{-1}, \quad \sigma_{\text {[oi] } 63 \mu \mathrm{m}} \sim 80-130 \mathrm{~km} \mathrm{~s}^{-1}\right.$; Contursi et al. 2013; Leroy et al. 2015), the LIRG ESO 320G030 $\left(\sigma_{\mathrm{CO}} \sim 30-70 \mathrm{~km} \mathrm{~s}^{-1}\right.$; Pereira-Santaella et al. 2016), and the AGN/LIRGs I12112 and I14348 $\left(\sigma_{\mathrm{CO}} \sim 30-120 \mathrm{~km} \mathrm{~s}^{-1}\right.$; Pereira-Santaella et al. 2018).

How to convert the flux densities associated with the outflow into molecular gas masses is still an open question. The CO-to- $\mathrm{H}_{2}$ conversion factor depends mainly on the metallicity and surface density of the gas (e.g., Bolatto et al. 2013b). In the case of the $\mathrm{CO}$ gas in the outflow, in the literature, there are three CO-to- $\mathrm{H}_{2}$ conversion factors commonly used: (1) a Galactic conversion factor of $\alpha_{\mathrm{CO}, \mathrm{MW}}=4.3 M_{\odot}\left(\mathrm{K} \mathrm{km} \mathrm{s}^{-1} \mathrm{pc}^{-2}\right)^{-1}$ (where $\mathrm{MW}$ stands for Milky Way; Bolatto et al. 2013b), (2) a ULIRG-like conversion factor of $\alpha_{\mathrm{CO}, \mathrm{ULIRG}}=0.8 M_{\odot}\left(\mathrm{K} \mathrm{km} \mathrm{s}^{-1} \mathrm{pc}^{-2}\right)^{-1}$ (e.g., Cicone et al. 2014; Feruglio et al. 2015; Veilleux et al. 2017), and (3) an optically thin conversion factor of $\alpha_{\mathrm{CO} \text {,thin }}=$ $0.34 M_{\odot}\left(\mathrm{K} \mathrm{km} \mathrm{s}^{-1} \mathrm{pc}^{-2}\right)^{-1}$ (e.g., Bolatto et al. 2013a; Richings \& Faucher-Giguère 2018). Assuming that the molecular gas in the outflows is optically thin-as observed, for example, in the jet-accelerated wind of IC 5063 (Dasyra et al. 2016) —would yield the most conservative (or "minimum") estimate of the molecular gas masses.

In this paper, we adopt the ULIRG-like conversion factor, which seems to be a good compromise, given the range of conversion factors available. In addition, the detection of dense molecular gas entrained in the outflow of starbursts and LIRGs (e.g., Aalto et al. 2012, 2015; Walter et al. 2017) argues in favor of a conversion factor higher than $\alpha_{\mathrm{CO}, \text { thin. }}$. In the main streamer of NGC 253, for example, the outflowing gas is not 

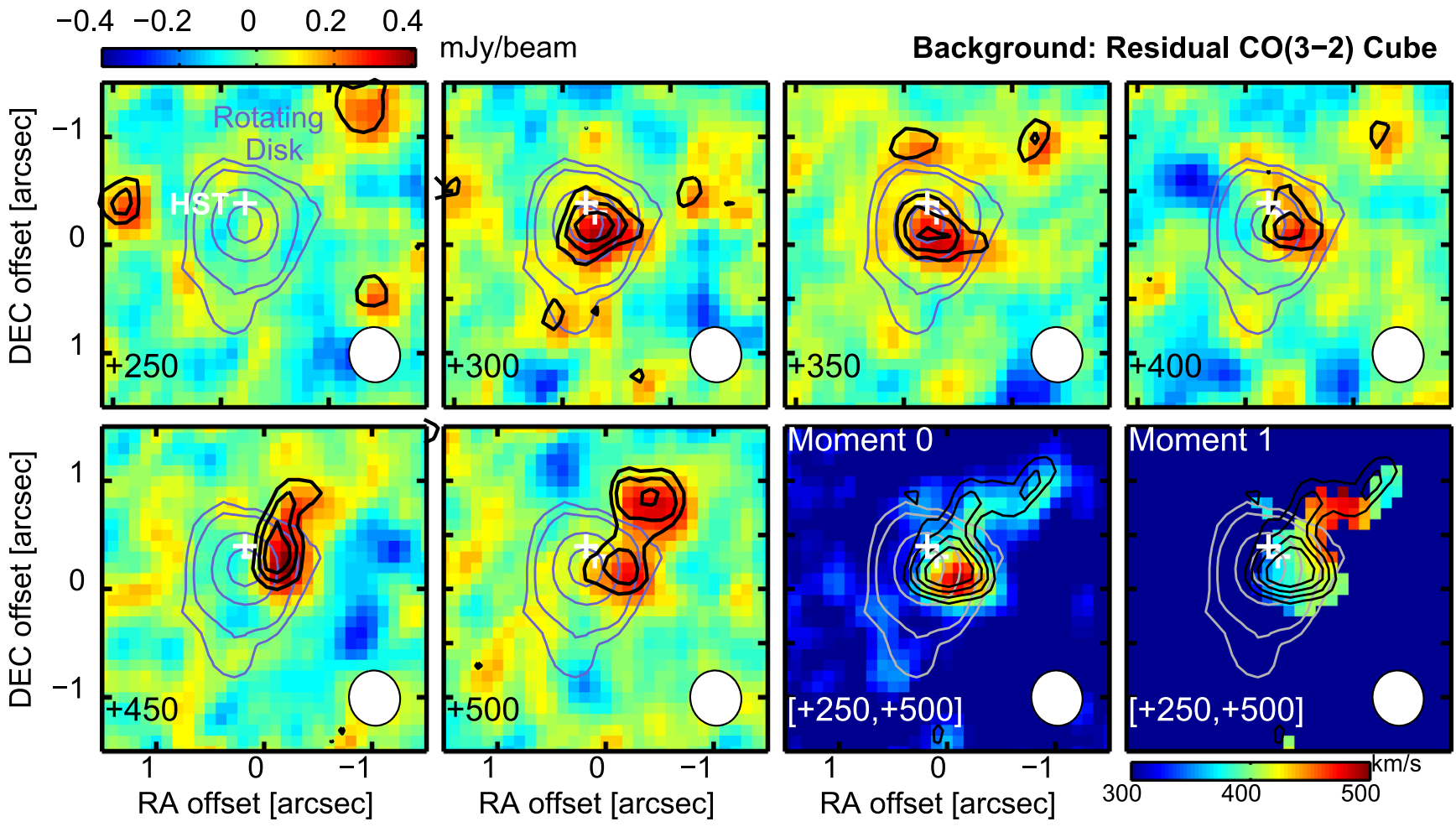

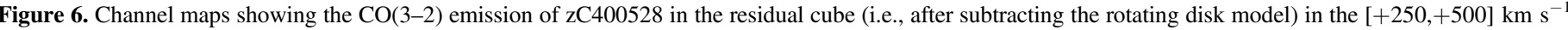

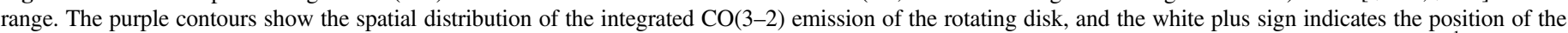

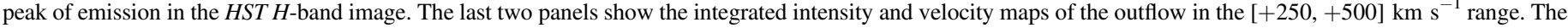
contours start at $2 \sigma$ and have increments of $1 \sigma$. The synthesized beam is shown in the bottom right corner.

optically thin, as the observed $\mathrm{CO}(2-1)$-to- $\mathrm{CO}(1-0)$ brightness temperature line ratio is about unity (Zschaechner et al. 2018). We also expect that the molecular gas in the outflow is likely not cold but warm, because it has been shocked at some level and/or is immersed in hot gas and subjected to a substantial external radiation field. In that case, the physical conditions of the molecular gas in the wind better resemble those found in the interstellar medium (ISM) of (U)LIRGs rather than the Milky Way.

The molecular gas masses for each of the outflow components are listed in Table 1. The total molecular gas mass in the outflow of $\mathrm{zC} 400528$ is

$$
M_{\text {out }, \text { mol }}=3.36 \times 10^{9} M_{\odot} \times\left(\frac{\alpha_{\mathrm{CO}}}{\alpha_{\mathrm{CO}, \mathrm{ULIRG}}}\right),
$$

which corresponds to $\sim 3 \%$ of the molecular gas mass in the disk. As Figure 8 shows, this fraction is similar to those measured in local (U)LIRG and Seyfert galaxies of similar stellar mass (Fiore et al. 2017 and references therein). ${ }^{23}$

\subsubsection{Molecular Mass Outflow Rate}

There are two common approaches to measure outflow mass rates in galaxies. They are often referred to as instantaneous (maximum) and average (minimum), and a detailed description

\footnotetext{
${ }^{23}$ Note that for the bodies of starbursts and (U)LIRGs drawn from the literature that are outliers in the local MS relation $\left(\Delta_{\mathrm{MS}} \gtrsim 1 \mathrm{dex}\right)$, we use the CO-to- $\mathrm{H}_{2}$ conversion factor from the scaling relation in Genzel et al. (2015) to calculate their molecular gas masses. This conversion factor is a factor of $\sim 2$ lower for starbursts and (U)LIRGs, depending on their specific star formation rate, and similar to that in Tacconi et al. (2018) for MS galaxies.
}

of the assumptions that go into the calculations can be found in Rupke et al. (2005). In the instantaneous approach, the outflow mass rate $\left(d M_{\text {out }} / d t\right)$ is calculated as the product of the outflow gas mass and the timescale taken by the gas to cross the thickness of the outflowing shell, i.e.,

$$
\dot{M}_{\text {out }}^{\text {inst }}=M_{\text {out }} \times \frac{v_{\text {out }}}{\Delta R},
$$

where $v_{\text {out }}$ is the velocity of the outflow and $\Delta R\left(=R_{\text {out }}-R_{\text {int }}\right)$ is the thickness of the outflow shell. This approach has been used in a number of studies, including Sturm et al. (2011) and González-Alfonso et al. (2014).

In the case of the average approach, the assumption is that the outflowing gas extends to $r=0$, so the outflow mass rate $\left(\dot{M}_{\text {out }}\right)$ is given by the outflow gas mass time-averaged over the flow timescale, i.e.,

$$
\dot{M}_{\text {out }}^{\text {avg }}=M_{\text {out }} \times \frac{v_{\text {out }}}{R_{\text {out }}} .
$$

If the emitting volume (spherical or multiconical) is filled with uniform density, then the mass outflow rate in Equation (2) should be a factor of 3 higher (e.g., Feruglio et al. 2010; Maiolino et al. 2012; Rodríguez Zaurín et al. 2013; Fiore et al. 2017).

In this paper, we use the average approach, which represents a more conservative way to characterize the outflow than the instantaneous method, as $\dot{M}_{\text {out }}^{\text {avg }}$ is smaller than $\dot{M}_{\text {out }}^{\text {inst }}$ by a factor $\left(R_{\text {out }} / \Delta R\right)$. Following this, the first step is to calculate the flow timescale, $t_{\text {out }}=R_{\text {out }} / v_{\text {out }}$. As described in Section 4.2.2, zC400528 has three main molecular outflow components. If we estimate the flow timescale based on the maximal extension of 

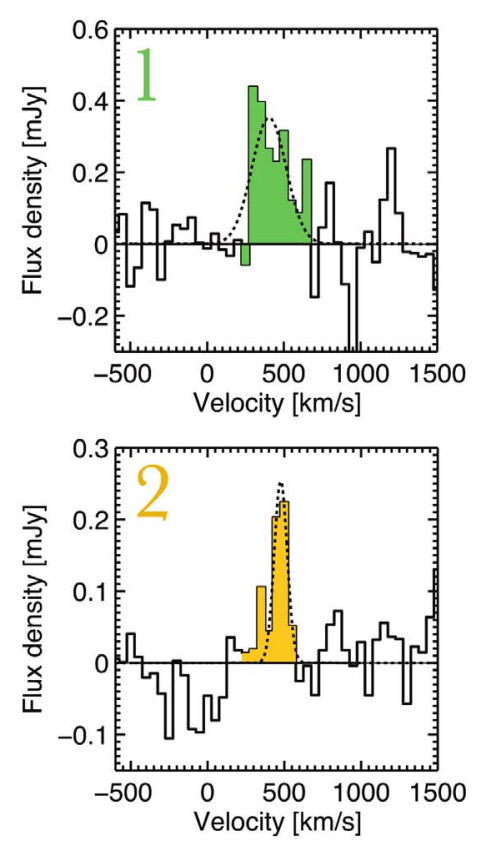
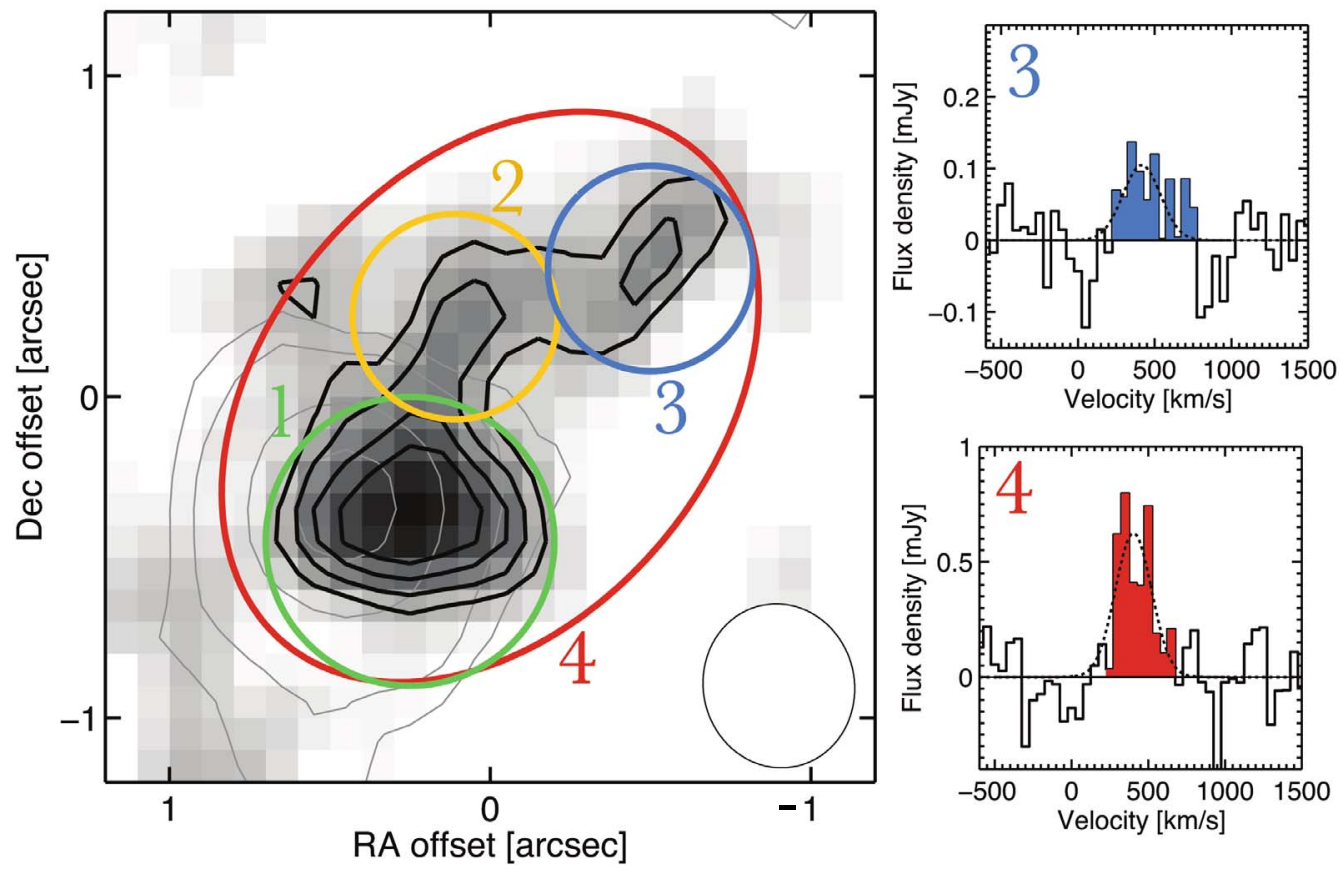

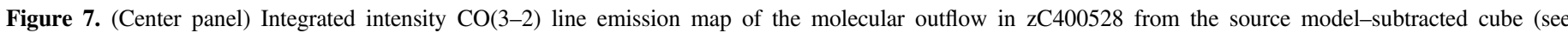

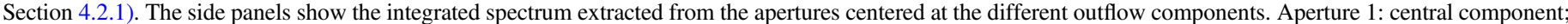

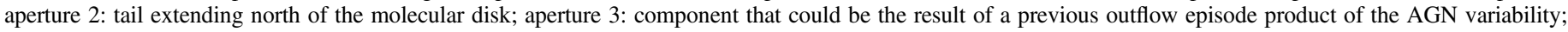

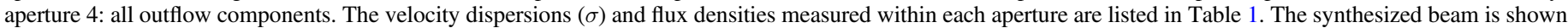
in the bottom right corner.

Table 1

Molecular Outflow Mass in zC400528

\begin{tabular}{lccc}
\hline \hline $\begin{array}{l}\text { Component } \\
\text { No. }\end{array}$ & $\begin{array}{c}\sigma \\
\left(\mathrm{km} \mathrm{s}^{-1}\right)\end{array}$ & $\begin{array}{c}\text { Integrated Flux } \\
\left(\mathrm{Jy} \mathrm{km} \mathrm{s}^{-1}\right)\end{array}$ & $\begin{array}{c}M_{\text {out, mol }}{ }^{\mathrm{a}} \\
\left(10^{9} M_{\odot}\right)\end{array}$ \\
\hline 1 & 158.0 & $0.098 \pm 0.016$ & $1.81(0.77-9.72)$ \\
2 & 60.0 & $0.029 \pm 0.007$ & $0.50(0.21-2.68)$ \\
3 & 173.5 & $0.032 \pm 0.007$ & $0.59(0.25-3.17)$ \\
\hline 4 & 167.0 & $0.184 \pm 0.029$ & $3.36(1.42-18.06)$ \\
\hline
\end{tabular}

Note.

${ }^{a}$ Molecular gas masses calculated using an $\alpha_{\mathrm{CO} \text {,ULIRG }}$ conversion factor. The values in parentheses correspond to the molecular gas masses we would obtain if we applied an $\alpha_{\mathrm{CO} \text {,thin }}$ or $\alpha_{\mathrm{CO} \text {,Mw }}$ conversion factor, respectively.

the outflow, then $t_{\text {out,mol }} \approx 8.5 \mathrm{kpc} / 400 \mathrm{~km} \mathrm{~s}^{-1} \approx 2 \times 10^{7} \mathrm{yr}$. A more representative or characteristic timescale would result from considering only the main outflow component (which corresponds to regions 1 and 2 in Figure 7). This component encompasses $\sim 80 \%$ of the outflow total mass and has a size of $\approx 4.2 \mathrm{kpc} \quad\left(R_{\text {out, } \mathrm{mol}} \approx 0 . \prime 5\right.$, according to a two-dimensional Gaussian fit and deconvolved from the beam) and a velocity of $\sim 450 \mathrm{~km} \mathrm{~s}^{-1}$. From these values, the resulting flow timescale is $t_{\text {out,mol }} \approx 4.2 \mathrm{kpc} / 450 \mathrm{~km} \mathrm{~s}^{-1} \approx 9 \times 10^{6} \mathrm{yr}$, which yields a mass outflow rate of

$$
\begin{aligned}
\dot{M}_{\mathrm{out}, \mathrm{mol}}^{\mathrm{avg}} \approx & 256 M_{\odot} \mathrm{yr}^{-1} \times\left(\frac{\alpha_{\mathrm{CO}, \text { out }}}{\alpha_{\mathrm{CO}, \mathrm{ULIRG}}}\right) \\
& \times\left(\frac{v_{\text {out }}}{450 \mathrm{~km} \mathrm{~s}^{-1}}\right) \times\left(\frac{4.2 \mathrm{kpc}}{R_{\mathrm{out}}}\right) .
\end{aligned}
$$

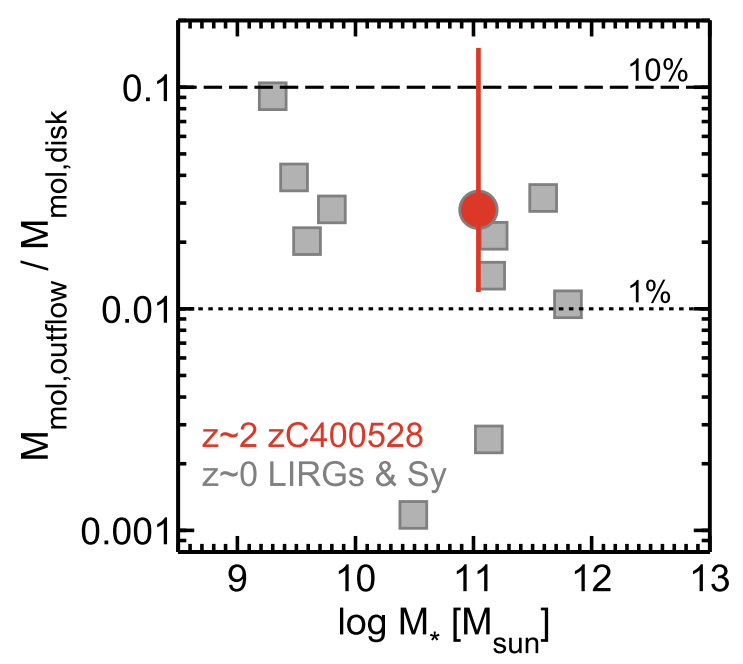

Figure 8. Ratio of the molecular gas mass of the AGN-driven outflow and the galaxy as a function of stellar mass for local (U)LIRGs and Seyferts (Fiore et al. 2017 and references therein) and our target, zC400528. In all cases, we assume an $\alpha_{\mathrm{CO} \text {,ULIRG }}$ conversion factor to estimate the molecular gas mass in the outflow. The vertical red line associated with the molecular gas fraction in zC400528 shows how the value would change if we assume an optically thin or MW CO-to- $\mathrm{H}_{2}$ conversion factor instead of $\alpha_{\mathrm{CO}, \mathrm{ULIRG}}$.

This translates into an outflow depletion time $\left(\tau_{\text {dep,out }}=\right.$ $\left.M_{\text {mol,disk }} / \dot{M}_{\text {out,mol }}^{\text {avg }}\right)$ of

$$
\begin{aligned}
t_{\text {dep }, \text { out }} \approx & 0.47 \mathrm{Gyr} \times\left(\frac{\alpha_{\mathrm{CO}, \text { disk }}}{\alpha_{\mathrm{CO}, \mathrm{T} 18}}\right) \times\left(\frac{\alpha_{\mathrm{CO}, \mathrm{ULIRG}}}{\alpha_{\mathrm{CO}, \text { out }}}\right) \\
& \times\left(\frac{450 \mathrm{~km} \mathrm{~s}^{-1}}{v_{\text {out }}}\right) \times\left(\frac{R_{\mathrm{out}}}{4.2 \mathrm{kpc}}\right) .
\end{aligned}
$$




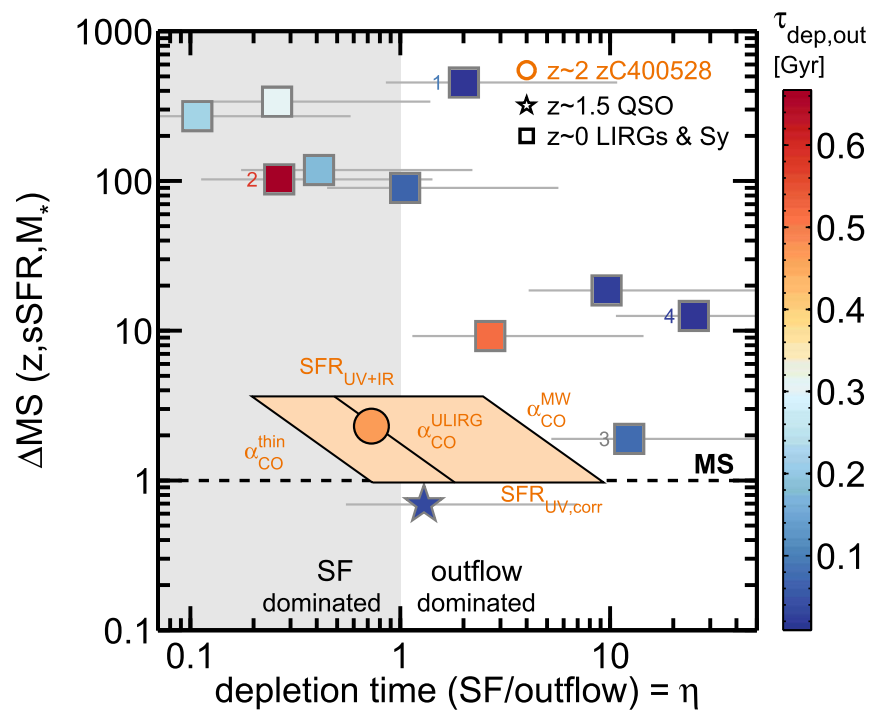

Figure 9. Separation from the MS of star-forming galaxies-after removing the dependence with redshift-vs. the ratio between the star formation and the outflow depletion timescales or, equivalently, the mass-loading factor ( $\eta=\dot{M}_{\text {out }} /$ SFR). In color, we show the outflow depletion timescale in Gyr. The colored area indicates the range of values covered by zC400528 (up and down limits if we use $\mathrm{SFR}_{\mathrm{UV}+\mathrm{IR}}$ or $\mathrm{SFR}_{\mathrm{UV} \text {,corr }}$ and left and right limits if we assume $\alpha_{\mathrm{CO} \text {,thin }}$ or $\alpha_{\mathrm{CO} \text {,Mw }}$ for the molecular gas in the outflow, respectively). The circle shows the position of $\mathrm{zC} 400528$ if we use the average of

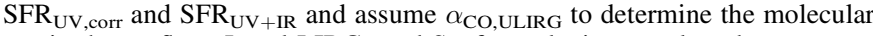
gas in the outflow. Local LIRGs and Seyfert galaxies are plotted as squares, and the $z=1.59$ QSO XID2028 is shown as a star (Brusa et al. 2018). The horizontal lines represent how much the molecular outflow masses increase or decrease if we assume $\alpha_{\mathrm{CO} \text {,thin }}$ or $\alpha_{\mathrm{CO}, \mathrm{MW}}$ instead of $\alpha_{\mathrm{CO} \text {,ULIRG }}$, respectively. The unshaded area shows where $\tau_{\text {dep,out }}<\tau_{\text {dep,SF}}$, i.e., where the outflow in the system is powerful enough to deplete the galaxy molecular gas reservoir in a timescale shorter than that needed to exhaust it by star formation. For reference, galaxies 1, 2, 3, and 4 are Mrk 231, NGC 6240, NGC 1433, and NGC 1068.

Relative to the star formation depletion timescale $\left(t_{\mathrm{dep}, \mathrm{SF}}=\right.$ $M_{\text {mol,disk }} / \mathrm{SFR}$ ), $t_{\text {dep,out }}$ is a factor of $\sim 2$ shorter or longer, depending on whether we consider the UV-corrected or UVplus-infrared SFR measurement of zC400528, respectively.

So far, we have ignored the effect that the inclination of the outflow has on the determination of the mass outflow rate and, consequently, the outflow depletion timescale. The reason is that we do not have enough information to constrain the geometry of the outflow. If $\theta$ corresponds to the inclination angle of the outflow with respect to the line of sight, then the deprojected outflow size is $R_{\text {out }}=R_{\text {out,proj }} / \sin (\theta)$, and the deprojected outflow velocity is $v_{\text {out }}=v_{\text {out,proj }} / \cos (\theta)$. This implies that the inclination-corrected mass outflow rate is proportional to $\tan (\theta)$. The inclination of $\mathrm{zC} 400528$ is $i \approx 37^{\circ}$ $(\sin (i)=0.61$; Förster Schreiber et al. 2018a). If we assume that the outflow is perpendicular to the disk, then the deprojection effects in velocity and radius almost cancel out, and the correction is small and of the order of $\sim 0.75$.

Figure 9 compares the outflow and star formation depletion timescales of galaxies as a function of their separation from the MS (after removing the dependence with $z$ using the Tacconi et al. 2018 scaling relations). The plot includes zC400528 and a compilation of local (U)LIRG and Seyfert galaxies (Fiore et al. 2017 and reference therein) and the $z=1.59$ QSO XID2028 (Brusa et al. 2018). We observe that local systems where the depletion of the molecular gas is dominated by star formation lie at least $\sim 1.5$ dex above the MS. On the other hand, galaxies where the depletion of molecular gas is dominated by outflows are located within $\sim 1$ dex of the MS, with the exception of the above outlier, Mrk 231 (Feruglio et al. 2010).

Similar to the local ULIRG/QSO Mrk 231 and the $z \approx 1.5$ QSO XID2028, zC400528 is exhausting its nuclear molecular gas reservoir via the outflow at a comparable rate, on average, at which stars are forming (keeping in mind the uncertainties and assumptions involved in the calculation). Note, however, the fundamental differences between these systems. While zC400528 represents a typical massive galaxy at $z \sim 2$, Mrk 231 lies a factor of $100 \times$ above the MS, and QSO XID2028, although in the MS, has a molecular gas depletion timescale of only $\sim 40-75$ Myr (Brusa et al. 2018). This is at least an order of magnitude lower than the typical depletion time for MS galaxies at a similar redshift (Tacconi et al. 2018). We discuss in more detail the effect that the powerful molecular outflow detected in zC400528 may have on quenching its star formation in Section 6.3.

\subsection{Energetics of the Molecular Outflow}

The molecular outflow in zC400528 could be driven by the AGN, the nuclear star formation activity, or, most likely, a combination of the two. If the dominant power source is the AGN, then the outflow will expand, conserving energy or momentum depending on how efficiently the inner quasirelativistic wind bubble $(v \sim 0.1 c)$ communicates its energy to the surrounding shocked gas.

In the energy-conserving case, the gas expands adiabatically, and most of the kinetic energy from the AGN wind is transmitted to the outflow. This results in large-scale $(\sim \mathrm{kpc}$ size) outflows with kinetic energies up to $\sim 2.5 \%$ of the AGN radiative power (assuming that half of the thermal energy goes into the bulk motion of the ISM) and momentum rates boosted to 5-20 times $L_{\mathrm{AGN}} / c$ (e.g., Faucher-Giguère \& Quataert 2012; Zubovas \& King 2012). In the momentum-driven case, the shocked expanding gas rapidly cools, and only the ram pressure of the wind is communicated to the outflow. This results in outflows that are confined to the nuclear region and have kinetic energies only up to $\sim 0.1 \%$ of the AGN luminosity and momentum rates of the order $\sim L_{\mathrm{AGN}} / c$ (e.g., King 2010; King \& Pounds 2015).

If the dominant power source for the outflow is star formation activity, then the combined momentum deposition of supernovae and stellar winds can supply a maximum momentum of ${ }^{24} \sim 2 \times L_{\mathrm{SF}} / c$ (where $L_{\mathrm{SF}}$ is the luminosity of the starburst) and inject a maximum mechanical energy of $\sim 0.2 \% \times L_{\mathrm{SF}}$ in the energy-conserving case (e.g., Murray et al. 2005; Veilleux et al. 2005).

By calculating the momentum flux $\left(\dot{P}_{\text {out }}=\dot{M}_{\text {out }} \times v_{\text {out }}\right)$ and energy flux $\left(\dot{E}_{\text {out }}=\frac{1}{2} \dot{M}_{\text {out }} \times v_{\text {out }}^{2}\right)$ of the molecular outflow in zC400528, we can explore if the AGN and/or the nuclear star formation activity can provide enough power to blow the gas and determine if the gas flow is energy- or momentumconserving.

\footnotetext{
${ }^{24}$ If we consider radiation pressure as an additional driving mechanism, then we need to add a contribution $\tau L_{\mathrm{SF}} / c$, where $\tau$ is the optical depth.
} 
From the mass outflow rate $\dot{M}_{\text {out }}^{\text {avg }}$ calculated in Section 4.2.4, the outflow momentum flux in $\mathrm{zC} 400528$ is

$$
\begin{aligned}
& \dot{P}_{\text {out } \text { mol }} \approx 7.2 \times 10^{35} \text { dynes } \times\left(\frac{\alpha_{\mathrm{CO}, \text { out }}}{\alpha_{\mathrm{CO}, \text { ULIRG }}}\right), \\
& \times\left(\frac{v_{\text {out }}}{450 \mathrm{~km} \mathrm{~s}^{-1}}\right)^{2} \times\left(\frac{4.2 \mathrm{kpc}}{R_{\text {out }}}\right)
\end{aligned}
$$

and the outflow kinetic energy flux is

$$
\begin{aligned}
\dot{E}_{\mathrm{out}, \mathrm{mol}} \approx & 1.6 \times 10^{43} \mathrm{erg} \mathrm{s}^{-1} \times\left(\frac{\alpha_{\mathrm{CO}, \text { out }}}{\alpha_{\mathrm{CO}, \text { ULIRG }}}\right) \\
& \times\left(\frac{v_{\text {out }}}{450 \mathrm{~km} \mathrm{~s}^{-1}}\right)^{3} \times\left(\frac{4.2 \mathrm{kpc}}{R_{\mathrm{out}}}\right) .
\end{aligned}
$$

We can now compare these quantities with the maximum estimated momentum and energy rates that the AGN and the star formation activity in zC400528 can provide. For this, we calculated the AGN bolometric luminosity $\left(L_{\mathrm{AGN}, \text { bol }}\right)$ using the rest-frame $7 \mu \mathrm{m}$ dust continuum emission (see the Appendix for details) and the star formation luminosity based on the SFR values following $L_{\mathrm{SF}}\left(L_{\odot}\right) \approx 10^{10} \times \operatorname{SFR}\left(M_{\odot} \mathrm{yr}^{-1}\right) \quad$ (e.g., Kennicutt \& Evans 2012).

Figure 10 shows the energetics of the molecular outflow in zC400528 compared to results from local (U)LIRGs (GonzálezAlfonso et al. 2017). The left and right panels depict the cases where we consider that the outflows are driven by AGN and star formation activity, respectively. In the AGN-driven case, the momentum boost in zC400528 is $\dot{P}_{\text {out }, \mathrm{mol}} /\left(L_{\mathrm{AGN}, \mathrm{bol}} / c\right) \approx 7$. This value is at the low end of the distribution of momentum boost found in local (U)LIRGs $\left(\dot{P}_{\text {out }} \sim 5-30 L_{\mathrm{AGN}, \text { bol }} / c\right.$; GonzálezAlfonso et al. 2017) but is high enough to consider the outflow as energy-conserving. This is consistent with the observed $\sim \mathrm{kpc}$ extent of the molecular outflow, as momentum-conserving outflows are expected to be confined to a small region (King \& Pounds 2015).

The outflow energy flux in zC400528 is $\dot{E}_{\text {out,mol }} \approx$ $0.5 \% L_{\mathrm{AGN}, \text { bol. }}$ This is a factor of $\sim 5$ below the maximum power that can be supplied by the AGN (shaded region) and could be the result of a low coupling efficiency of the wind with the ISM. We conclude that the AGN activity in zC400528 is sufficient to explain the observed momentum boost and kinetic power of the molecular outflow.

In the case where we consider the star formation-driven scenario (right panels in Figure 10), we observe that star formation activity can only provide sufficient energy to power the outflow if the star formation rate of $\mathrm{zC} 400528$ is close to the $\mathrm{SFR}_{\mathrm{UV}+\mathrm{IR}}$ value. Remember that the latter is most likely an upper limit to the SFR, as it may include contributions from AGN activity and heating of the dust by evolved stars (see Section 2).

In summary, the most likely scenario is that the molecular outflow in zC400528 is powered by a combination of nuclear star formation and AGN activity, with a dominant contribution from the latter. The molecular outflow mass and energetics measured in zC400528 for different assumptions on $\alpha_{\mathrm{CO}}$ can be found in Table 2 .

\section{Comparison between Molecular and Ionized Outflow}

Our current knowledge of the multiphase structure and energetics of galactic outflows at high- $z$ remains greatly limited by the scarcity of observations that target both the ionized and neutral phases of the outflow. The subject of this study, zC400528, represents an exception, with high-resolution observations of the ionized (VLT/SINFONI adaptive opticsassisted) and molecular (ALMA) outflow gas available. In this section, we use these complementary data sets to compare the spatial distribution and energetics of the ionized and molecular phases of the outflow.

\subsection{Morphology}

Figure 11 shows the spatial distribution of the molecular disk (background), the $\mathrm{H} \alpha$ blue and red broad (outflow) component (green), and the molecular wind (red). The bulk of the projected $\mathrm{H} \alpha$ broad emission extends $\sim 4 \mathrm{kpc}$ north from the nuclear region. Beyond this point, the only two extranuclear ionized outflow components detected, A and B, are cospatial with the molecular outflow gas, and-at least in the case of region A, as Figure 12 shows - the molecular and ionized gas velocities are similar. The velocity dispersions extracted from the Gaussian fit to the spectra of regions A and B are comparable with those observed in the spatially resolved molecular outflows of local starbursts and (U)LIRGs (see Section 4.2.3; Contursi et al. 2013; Leroy et al. 2015; Pereira-Santaella et al. 2016) and appear to be higher than those measured in the tidal tails of interacting LIRGs $\left(\sigma_{\mathrm{CO}} \sim 15 \mathrm{~km} \mathrm{~s}^{-1}\right.$; Pereira-Santaella et al. $2018)$ or in the Antennae $\left(\sigma_{\mathrm{H}} \sim 5-15 \mathrm{~km} \mathrm{~s}^{-1}\right.$; Hibbard et al. 2001). With the current data, however, it is difficult to rule out the possibility that regions $\mathrm{A}$ and $\mathrm{B}$ are part of a tidal tail or a second merger galaxy.

Similar to zC400528, Cresci et al. (2015) and Brusa et al. (2018) found that the ionized and molecular phases of the outflow in the QSO XID2028 $(z=1.59)$ extend for about $\sim 8 \mathrm{kpc}$ over the same spatial region. This is consistent with the scenario where AGNs with high-duty cycles on massive galaxies can drive ionized and molecular outflows over scales of tens of kpc (see Section 6.2).

The spatial distribution of the outflow in $\mathrm{zC} 400528$ suggests that the ionized gas is outflowing inclined $\left(\sim 60^{\circ}\right)$ with respect to the major kinematic axis, likely along the path of least resistance outside the galaxy plane. There are two main molecular outflow components in the inner $\sim 4 \mathrm{kpc}$ region: one that extends alongside the ionized wind (from the center to region $\mathrm{A}$ ) and one that is aligned with the major kinematic axis and could therefore be equatorial. This picture resembles the structure of the AGN-driven outflow in NGC 1068 (Tecza et al. 2001; Cecil et al. 2002). There, the ionized cone is inclined $\sim 40^{\circ}$ with respect to the galaxy disk, and the molecular outflow extends preferentially along the galaxy plane launched as a result of the ionization cone sweeping the molecular gas in the inner disk (García-Burillo et al. 2014). The fact that the outflow in zC400528 appears to be not perpendicular to the disk is a common feature observed in AGN-driven outflows. Star formation-driven outflows, on the other hand, tend to align with the minor kinematic axis of the disk (e.g., Leroy et al. 2015; Pereira-Santaella et al. 2018).

The configuration of the ionized and molecular phases of the outflow in $\mathrm{zC} 400528$ is also in qualitative agreement with the modeling work of Zubovas \& Nayakshin (2014). They find that 
AGN-driven

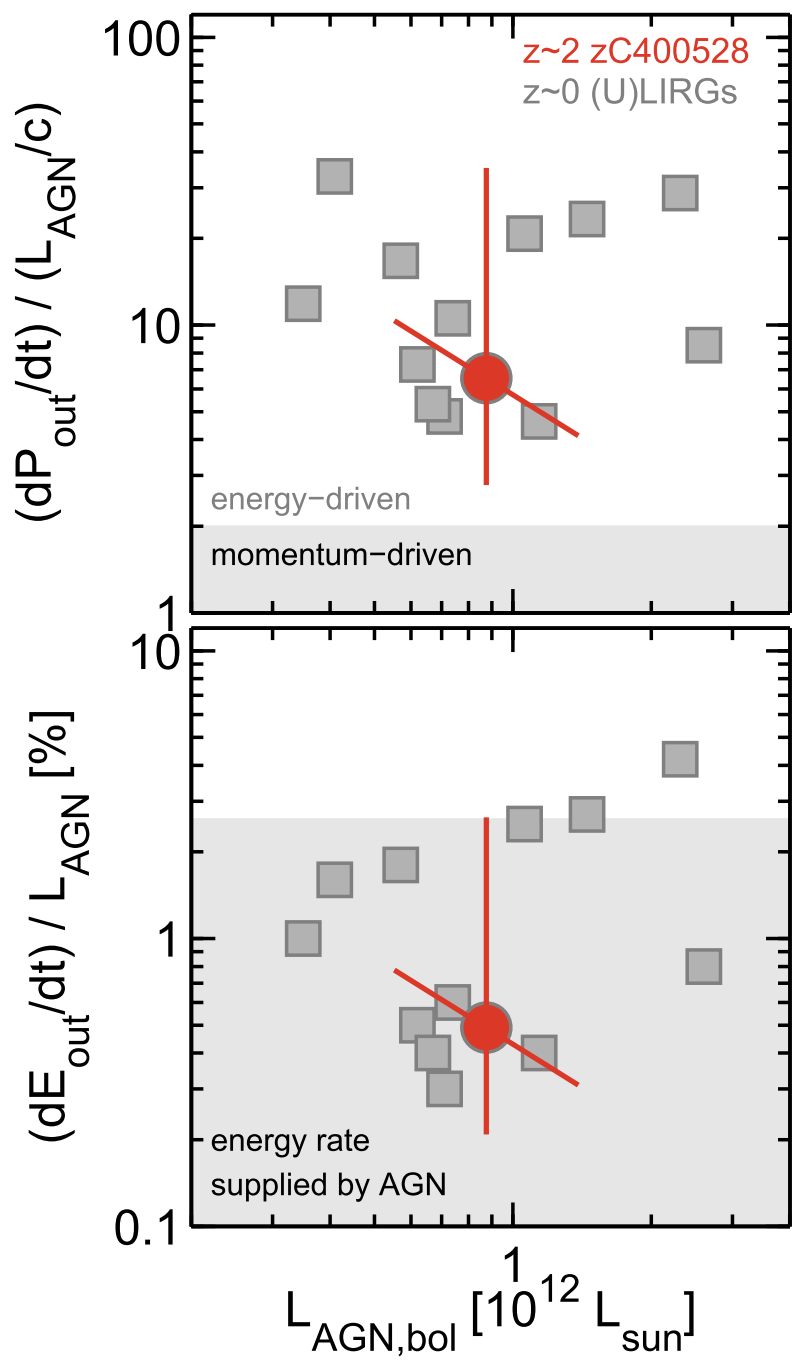

Star formation-driven

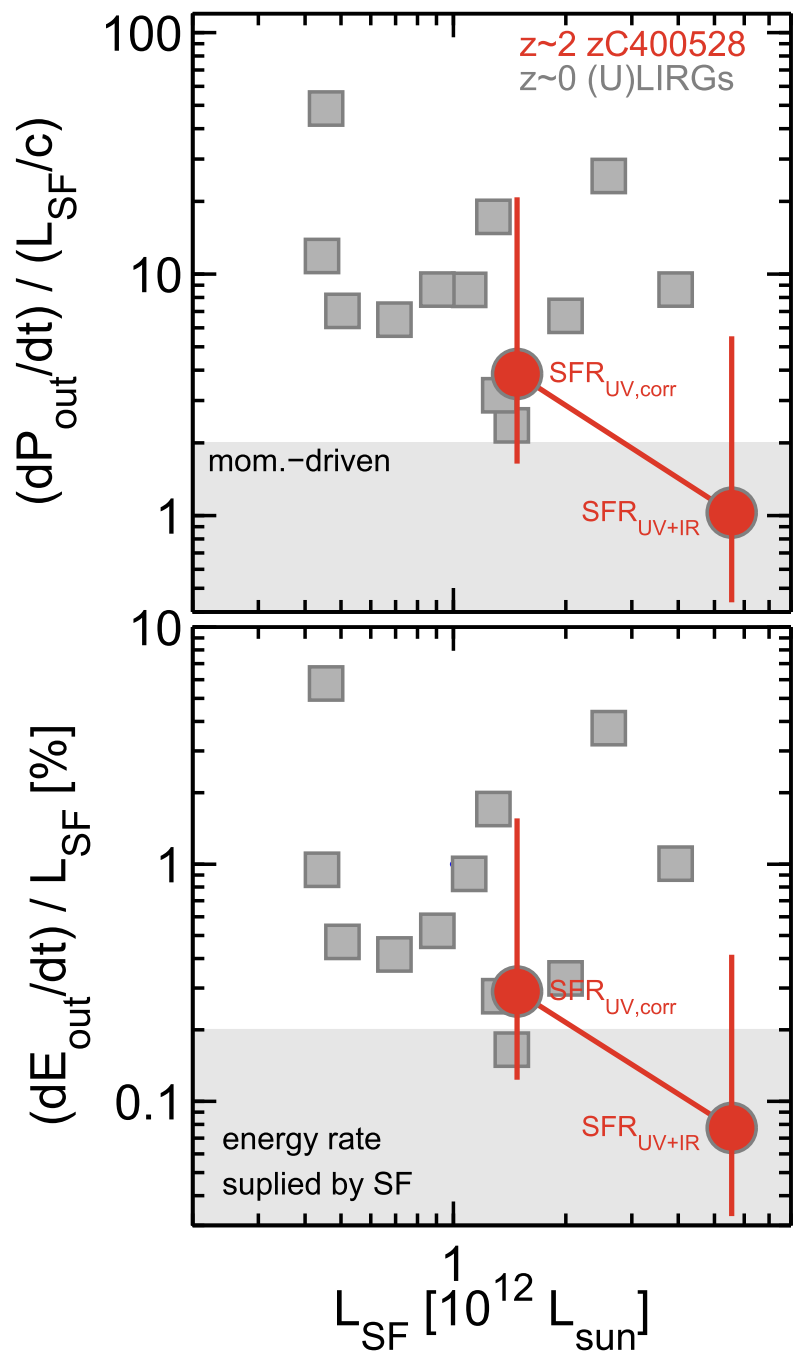

Figure 10. Molecular outflow energetics of zC400528 compared to (U)LIRGs from González-Alfonso et al. (2017). We consider possible sources for the outflow AGN (left) and star formation activity (right). The top panels show the outflow momentum flux ( $\left.\dot{p}_{\text {out }}\right)$ normalized by the radiation momentum rate as a function of the luminosity produced by the AGN ( $L_{\mathrm{bol}, \mathrm{AGN}} / c$ and $L_{\mathrm{bol}, \mathrm{AGN}}$; left) and the star formation activity $\left(L_{\mathrm{SF}} / c\right.$ and $L_{\mathrm{SF}}$; right). The bottom panels show the outflow mechanical power $\left(\dot{E}_{\text {out }}\right)$ normalized by the luminosity produced by the AGN (left) and the starburst (right). The shaded rectangles mark the momentum and energy rates that can be supplied by an AGN and the star formation activity. In the case of $\mathrm{zC} 400528$, we show the expected values when assuming SFR $\mathrm{UV}_{\text {,corr }}$ and $\mathrm{SFR} \mathrm{UV}_{+\mathrm{IR}}$. The vertical lines show how the values change if we assume an optically thin or MW CO-to- $\mathrm{H}_{2}$ conversion factor instead of $\alpha_{\mathrm{CO}, U L I R G}$.

AGN-driven outflows that start spherical quickly develop a bipolar morphology that expands faster and further in the polar direction (the direction of least resistance) than in the equatorial direction. After $\sim 10 \mathrm{Myr}$ (the flow timescale in zC400528), several cold dense clumps have formed embedded in the hot gas and are moving upward, while in the plane of the galaxy, the cold gas, squeezed by the expanding bubbles, is being pushed outward with a mean velocity of $\sim 400 \mathrm{~km} \mathrm{~s}^{-1}$.

\subsection{Mass and Energetics}

The ionized mass in the outflow of $\mathrm{zC} 400528$ is $M_{\text {out,ion }}=$ $2.2 \times\left(300 \mathrm{~cm}^{-3} / n_{\mathrm{e}}\right) \times 10^{8} M_{\odot}$ (Genzel et al. 2014), where $n_{\mathrm{e}}$ corresponds to the electron density of the ionized gas in the outflow. Recent results by Förster Schreiber et al. (2018b) find that the mean electron density in the outflow gas of $z \sim 2$ galaxies is $n_{\mathrm{e}} \sim 350 \mathrm{~cm}^{-3}$.

For an outflow size of $R_{\text {out,ion }}=3 \mathrm{kpc}$ (Förster Schreiber et al. 2014) and an outflow velocity of $v_{\text {out,ion }}=802 \mathrm{~km} \mathrm{~s}^{-1}$
(Genzel et al. 2014), the ionized mass outflow rate is $\dot{M}_{\text {ion,out }}=53 M_{\odot} \mathrm{yr}^{-1}$. Based on the same assumptions, the ionized momentum and energy outflow rates are $\dot{P}_{\text {out,ion }}=2.7 \times$ $10^{35}$ dynes and $\dot{E}_{\text {out,ion }}=1.1 \times 10^{43} \mathrm{erg} \mathrm{s}^{-1}$, respectively. Figure 13 summarizes the mass and mass-loss rates measured in the molecular and ionized outflow gas in zC400528. The molecular phase dominates the total observed budget of both $M_{\text {out }}$ and $\dot{M}_{\text {out }}$. Note, however, that there are gas phases in the outflow that remain unobserved and could change the overall balance, including very hot gas that could contribute to the ionized outflow mass (e.g., Veilleux et al. 2005).

A summary of the ionized outflow mass and energetics measured in zC400528 for different assumptions of $n_{\mathrm{e}}$ and the comparison to the quantities measured in the molecular phase can be found in Table 2 .

Another quantity of interest in the study of galactic outflows is the mass-loading factor $\left(\eta_{\text {phase }}\right)$, which is defined as the ratio between the mass outflow rate and the SFR. Figure 14 shows 
Table 2

Molecular and Ionized Gas Outflow Masses and Energetics

\begin{tabular}{|c|c|c|c|c|}
\hline Phase & $\begin{array}{c}\text { Mass } \\
10^{9} M_{\odot}\end{array}$ & $\underset{M_{\odot} \mathrm{yr}^{-1}}{\dot{y}_{\text {out }}}$ & $10^{35} \stackrel{\dot{P}_{\text {out }}}{\text { dynes }}$ & $10^{43} \dot{E}_{\text {out }} \mathrm{erg} \mathrm{s}^{-1}$ \\
\hline Molecular $^{\mathrm{a}}$ & $3.36(1.42-18.06)$ & $256(108.8-1376.0)$ & $7.2(3.1-38.7)$ & $1.6(0.68-8.6)$ \\
\hline Ionized $^{\mathrm{b}}$ & $0.22(0.07-0.66)$ & $53(15.9-159.0)$ & $2.7(0.81-8.1)$ & $1.10(0.33-3.30)$ \\
\hline
\end{tabular}

Notes.

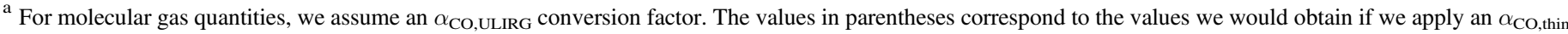
or $\alpha_{\mathrm{CO}, \mathrm{Mw}}$ conversion factor, respectively.

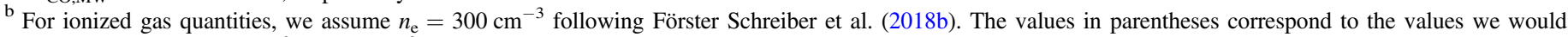
obtain if we assume $n_{\mathrm{e}}=10^{3}$ or $100 \mathrm{~cm}^{-3}$, respectively.

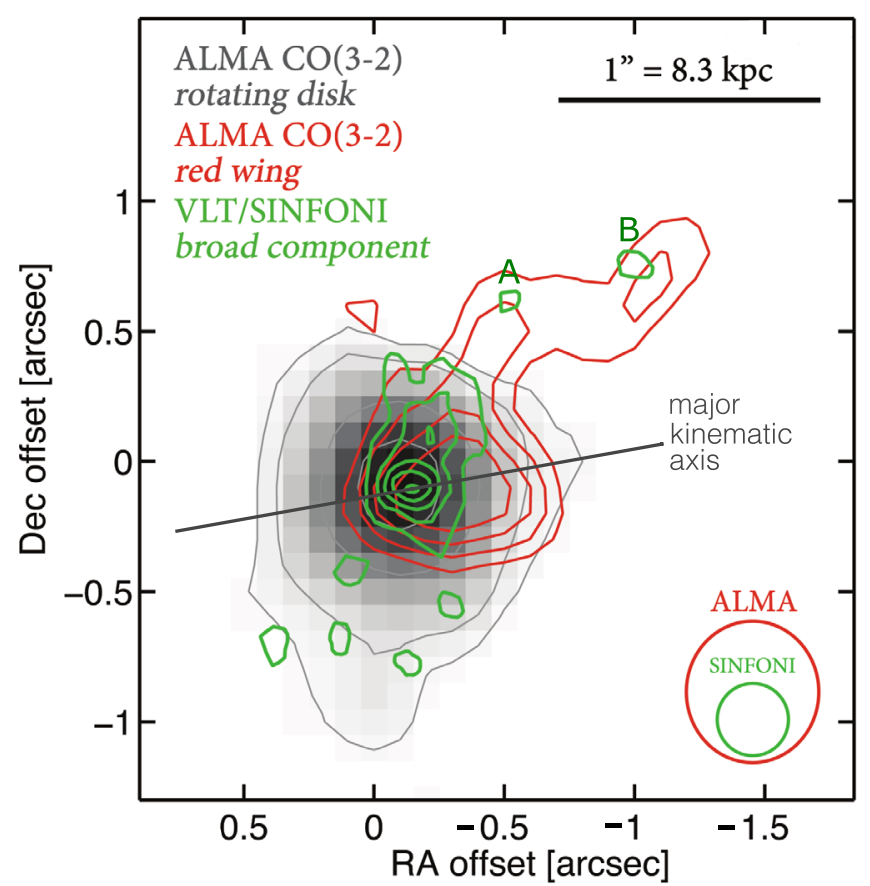

Figure 11. Spatial distribution of the molecular (red) and ionized (green; Förster Schreiber et al. 2014) outflow components of zC400528 overlaid on a map of its molecular disk as traced by the ALMA CO(3-2) data. The gray solid line shows the orientation of the kinematic major axis, the black horizontal bar shows the spatial scale, and the ellipses in the lower right corner show the angular resolution achieved by the high-resolution SINFONI+AO (Förster Schreiber et al. 2018a) and ALMA observations. The letters A and B are assigned to the extranuclear regions $(\gtrsim 4 \mathrm{kpc})$ in the outflow that are detected in both molecular and ionized gas. As Figure 12 shows, the central velocities of the $\mathrm{CO}$ and $\mathrm{H} \alpha$ lines in these extranuclear regions are comparable.

the molecular and ionized mass-loading factors as a function of the separation from the MS (removing the $z$ dependence using the Tacconi et al. 2018 scaling relations) for nearby starbursts and AGN galaxies (Heckman et al. 2015; Fiore et al. 2017) and massive $\left(\log \left(M_{*} / M_{\odot}\right) \geqslant 10.9\right)$ star-forming galaxies at $z \sim 1-3$ (this work; Genzel et al. 2014). The ionized mass-loading factors of these high- $z$ galaxies ${ }^{25}$ are found to be comparable to those observed in local MS outliers. The ionized and mass-loading factors in zC400528 are also similar to those found in the $z \sim 1.5$ QSOs XID2028 $\left(\eta_{\mathrm{mol}} \sim 1.3\right.$ and $\eta_{\text {ion }} \gtrsim 1$; Cresci et al. 2015; Brusa et al. 2018) and 3C $298\left(\eta_{\text {mol }} \sim 2.5\right.$ and $\eta_{\text {ion }} \sim 1.6$; Vayner et al. 2017).

\footnotetext{
${ }^{25}$ The ionized gas outflow masses reported in Genzel et al. (2014) are calculated assuming $n_{\mathrm{e}}=80 \mathrm{~cm}^{-3}$ in the ionized outflowing gas. Here we rescaled those values to match our assumption of $n_{\mathrm{e}}=300 \mathrm{~cm}^{-3}$.
}

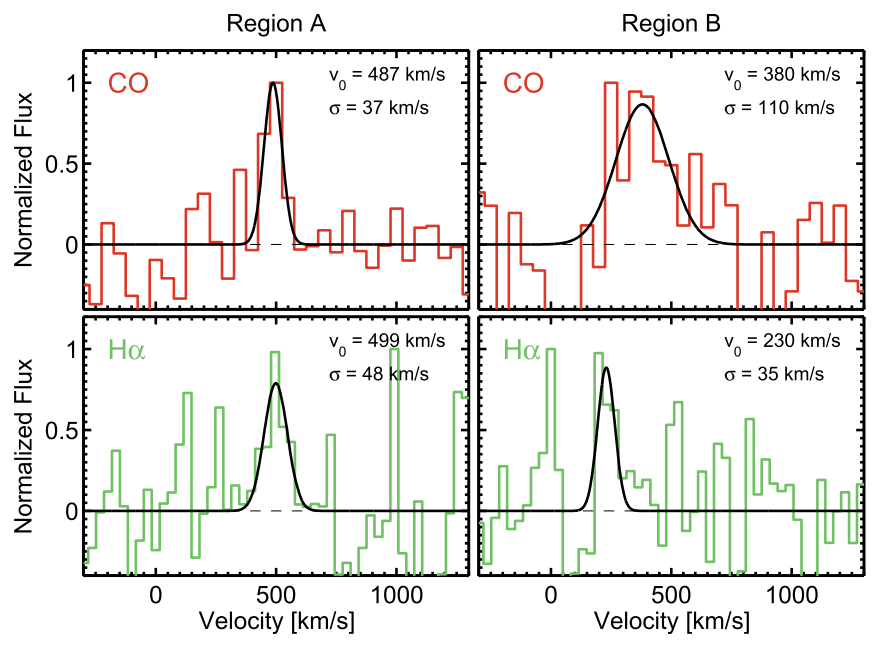

Figure 12. The $\mathrm{CO}(3-2)$ (red) and $\mathrm{H} \alpha$ (green) spectra extracted using $\approx 0{ }^{\prime \prime} 5$ apertures centered on extranuclear regions A and B (see Figure 11) in the outflow of zC400528. The Gaussian fit to the lines is shown in black, and the fitted central velocity and line width $(\sigma)$ values are listed in the top right corner of each panel.

The molecular mass outflow rate of zC400528 is comparable to some of the powerful molecular outflows observed in local starbursts and Seyfert galaxies (see also Section 4.2.4). In addition, the molecular mass-loading factor is $\sim 4$ times higher than its ionized counterpart. Note, however, that these results depend on assumptions of the properties of the outflow gas that are not yet fully constrained (e.g., the CO-to- $\mathrm{H}_{2}$ conversion factor, electron density, geometry, etc.). The same caveat applies to all of the other measurements shown in the figure.

\section{Discussion}

\subsection{Can the Outflowing Material Escape the Gravitational Potential of Its Host?}

Understanding whether baryons ejected by winds escape the galaxy or rain back onto the disk is key to reproducing the observed galaxy stellar masses and the metal enrichment of the intergalactic medium (IGM; e.g., Oppenheimer et al. 2010; Muratov et al. 2015). Simulations show that about half of the ejected outflow mass across all galaxy masses is later reaccreted (Übler et al. 2014; Christensen et al. 2016), and that in massive $z \sim 2$ galaxies, about $\sim 30 \%$ of the stellar mass forms from gas contributed by wind recycling (Anglés-Alcázar et al. 2017).

To determine if the molecular gas in the outflow of zC400528 can permanently escape the galaxy, we first estimate the escape velocity $\left(v_{\mathrm{esc}}\right)$ from the host. For this, we follow 

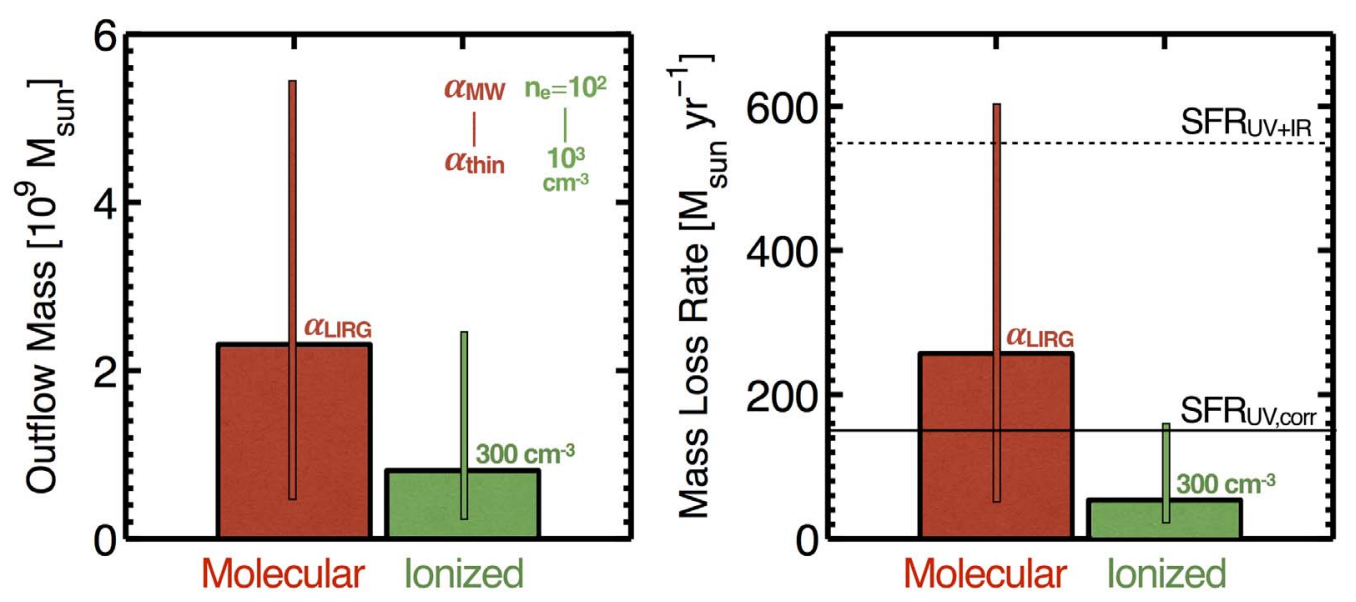

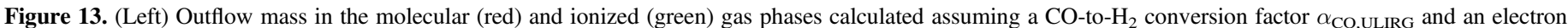

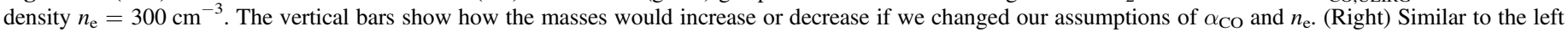

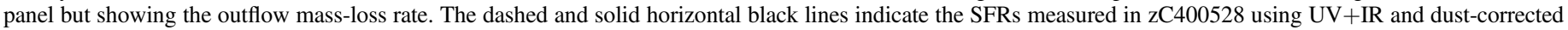
UV emission, respectively.

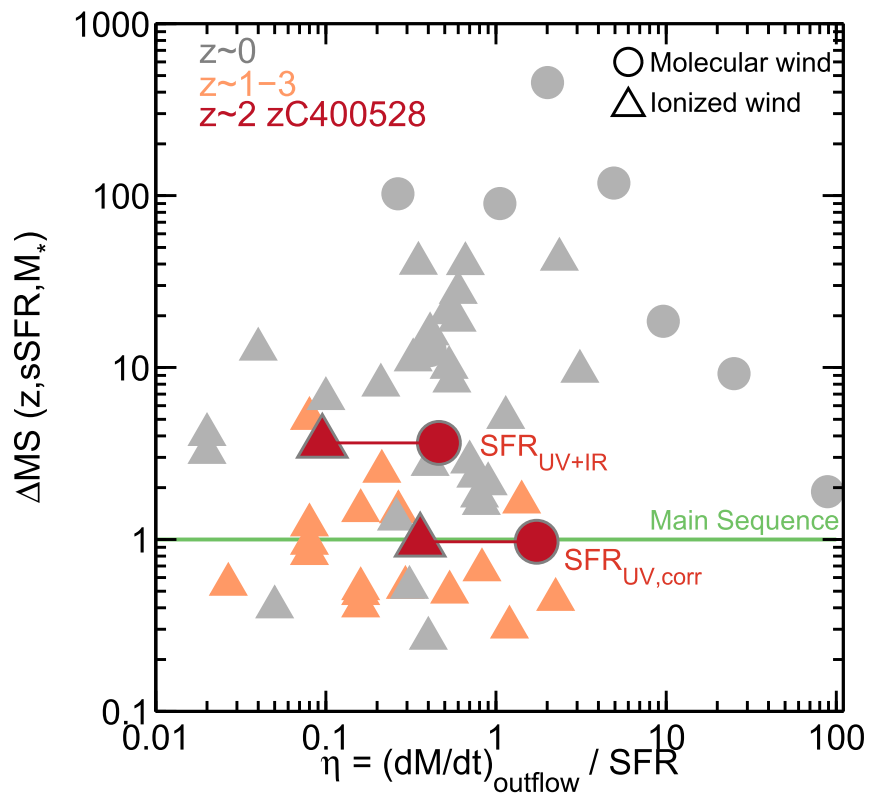

Figure 14. Separation from the MS of galaxies (after removing the dependence with redshift) vs. their molecular and ionized mass-loading factors ( $\eta \equiv \dot{M}_{\text {out }} /$ SFR). The circles and triangles show the results for molecular and ionized winds, respectively. In the case of $\mathrm{zC} 400528$, the molecular (this work) and ionized (Förster Schreiber et al. 2014; Genzel et al. 2014) outflow components are shown in red. We include the expected values when assuming $\mathrm{SFR}_{\mathrm{UV} \text {,corr }}$ and $\mathrm{SFR}_{\mathrm{UV}+\mathrm{IR}}$. The horizontal red line shows how the molecular gas quantities change if we assume an optically thin or MW CO-to- $\mathrm{H}_{2}$ conversion factor instead of $\alpha_{\mathrm{CO} \text {,ULIRG. }}$. We also include massive star-forming galaxies in the redshift range $z \sim 1-3$ from Genzel et al. (2014) and nearby starburst and AGN galaxies from Heckman et al. (2015) and Fiore et al. (2017).

Rupke et al. (2002) and use a simple gravitational model based on a truncated $\left(r<r_{\max }\right)$ isothermal sphere, so $v_{\mathrm{esc}}(r)=$ $\sqrt{2} v_{\text {circ }}\left[1+\ln \left(r_{\max } / r\right)\right]^{1 / 2} \cdot{ }^{26}$ Assuming that the dark matter halo extends to $r_{\max } \sim 100 \mathrm{kpc}$, and using a circular velocity for zC400528 of $v_{\text {circ }}=344 \mathrm{~km} \mathrm{~s}^{-1}$ (Förster Schreiber et al. $2018 \mathrm{a}$ ), the escape velocity at $r=10 \mathrm{kpc}$ is $v_{\mathrm{esc}, 10 \mathrm{kpc}} \sim 880$ $\mathrm{km} \mathrm{s}^{-1}$. The highest-velocity molecular outflow material we detect in zC400528 has a velocity of $v_{\text {out }} \approx 500 \mathrm{~km} \mathrm{~s}^{-1}$. If we

26 The escape velocity is only weakly sensitive to the exact value of $r_{\max } / r$. take inclination effects into account (see Section 4.2.4), the deprojected outflow velocity could increase to $v_{\text {out }} \sim 650 \mathrm{~km} \mathrm{~s}^{-1}$, which is still lower than the escape velocity. This suggests that the bulk of the expelled molecular gas mass will be reaccreted back onto the galaxy on timescales-according to simulations-no shorter than 1-3 Gyr (Oppenheimer et al. 2010; Übler et al. 2014; Christensen et al. 2016; Brennan et al. 2018).

In the case of the ionized outflowing gas, the escape velocity at $3 \mathrm{kpc}-\mathrm{a}$ distance about the size of the ionized outflowis $v_{\mathrm{esc}, 3 \mathrm{kpc}} \sim 1000 \mathrm{~km} \mathrm{~s}^{-1}$. The highest-velocity gas in the ionized wind can reach velocities up to $\sim 800 \mathrm{~km} \mathrm{~s}^{-1}$ (Genzel et al. 2014), which, if we correct by inclination, can increase to $\sim 1000 \mathrm{~km} \mathrm{~s}^{-1}$ and potentially escape the galaxy.

The very small fraction of the molecular and ionized outflow gas that can escape the gravitational field of zC400528 is consistent with the low escape fractions $(\lesssim 20 \%)$ measured in neutral, ionized, and molecular gas winds in local (U)LIRGs (e.g., Rupke et al. 2005; Arribas et al. 2014; Fluetsch et al. 2019; Pereira-Santaella et al. 2018).

\subsection{AGN Flickering and Outflow Expansion}

Observational and theoretical arguments suggest that the AGN lifetime of $\sim 10^{7}-10^{9} \mathrm{yr}$ is, in reality, a succession of hundreds or thousands of short $\left(\sim 10^{5} \mathrm{yr}\right)$ phases of supermassive black hole growth (e.g., Schawinski et al. 2015) —a process called AGN flickering. These periods of episodic AGN activity inflate large-scale outflows that can continue to expand even after the AGN finally switches off as a result of clearing the gas in the central region.

Zubovas \& King (2016) used an analytical model to follow the evolution of the molecular and ionized phases in an outflow driven by an AGN that "flickers" on timescales of $5 \times 10^{4} \mathrm{yr}$. They found that the oscillations caused by the AGN flickering smooth out relatively fast ( $\sim 3 \mathrm{Myr}$ ), so from an observational standpoint, it would appear that the outflow is driven by an AGN with constant luminosity. For a massive galaxy similar to $\mathrm{zC} 400528\left(M_{\mathrm{h}} \approx 10^{12.5-13} M_{\odot}\right.$, based on the relation between halo and stellar mass in Moster et al. 2013), the model predicts that in the outflow timescale of $\mathrm{zC} 400528\left(\sim 10^{7} \mathrm{yr}\right)$, the wind should have expanded between $\sim 4$ and $8 \mathrm{kpc}$ with expansion velocities at that distance of $\sim 300$ and $700 \mathrm{~km} \mathrm{~s}^{-1}$ for AGN 
duty cycles of $f_{\mathrm{AGN}}=20 \%$ and $100 \%$, respectively. The observed extent $(\sim 8 \mathrm{kpc})$ and velocity $\left(\sim 500 \mathrm{~km} \mathrm{~s}^{-1}\right)$ in the molecular outflow of zC400528 is consistent with these model results and favors the scenario with a high AGN duty cycle, in agreement with the results of Genzel et al. (2014) and Förster Schreiber et al. (2018b).

\subsection{Is zC400528 Moving Down from the MS of Star-forming Galaxies to Join the Population of Compact Passive Galaxies?}

There is growing evidence that suggests that when massive star-forming galaxies at $z \approx 2$ reach central stellar surface densities similar to those observed in massive ellipticals at $z \approx 0\left(\Sigma_{\star} \gtrsim 10^{10} M_{\odot} \mathrm{kpc}^{-2}\right.$ inside the inner $\sim \mathrm{kpc}$ region $)$, an effective quenching mechanism must act on relatively short timescales to shut down the star formation activity. Feedback from AGNs, prevalent in massive $z \sim 2$ galaxies (e.g., Genzel et al. 2014; Förster Schreiber et al. 2018b), is one of the obvious quenching candidates. In the specific case of zC400528, Tacchella et al. (2015a) suggested that the star formation activity in the inner $\sim 3 \mathrm{kpc}$ region should be fully quenched on a $\lesssim 0.5$ Gyr timescale.

To evaluate if $\mathrm{zC} 400528$ is on the path of significantly suppressing its star formation activity, we compare the timescales of depletion $\left(t_{\mathrm{dep}}\right)$ and replenishment $\left(t_{\mathrm{rep}}\right)$ of cold gas. For a galaxy to experience a long-term episode of quenching, we should crudely expect that $t_{\text {rep }} / t_{\text {dep }} \gtrsim 10$ (e.g., Tacchella et al. 2016).

From the analysis of the molecular outflow (Section 4.2.4) and star formation activity (Tacchella et al. 2018) in zC400528, we know that the outflow and star formation depletion times are $t_{\text {dep,out }} \sim t_{\text {dep,SF }} \sim 0.5 \mathrm{Gyr}$. These timescales can be a factor of $\sim 3$ shorter if we only consider the molecular gas inside the inner $\sim 4 \mathrm{kpc}$ region. Regarding the replenishment of gas, according to the prescription in Bouché et al. (2010; and ignoring the supply of gas provided by potential merger episodes; see also Lilly et al. 2013), a massive galaxy like zC400528 at $z \sim 2$ should accrete $\dot{M}_{\text {gas,in }} \sim 240 M_{\odot} \mathrm{yr}^{-1}$ of fresh gas. ${ }^{27}$ At this rate, and in the absence of strong preventive feedback, the inflow of fresh and recycled gas into the system should be enough to roughly maintain the level of star formation activity (i.e., $t_{\text {rep }} \sim 1-3 \times t_{\text {dep }}$ ). This is consistent with the results from the cosmological zoom-in simulations by Brennan et al. (2018) that follow the evolution of massive, gasrich galaxies $\left(M_{\star} \sim 10^{11} M_{\odot}\right.$ and $M_{\text {cold-gas }} / M_{\star} \sim 0.5-1$ at $z \approx 2$ ) from $z \approx 4$ to $z=0$. For the simulated galaxies that only include stellar and supernova feedback, Brennan et al. (2018) found that over the galaxy's lifetime, there is an overall balance between the inflow of gas and the level of star formation activity.

If AGN activity is considered, ${ }^{28}$ Brennan et al. (2018) showed that this equilibrium drastically changes. As AGN feedback starts to kick in at $z \sim 2$, the inflow rate of fresh gas

\footnotetext{
27 To calculate the inflow rate, we use the expression for the mean gas accretion rate in Bouché et al. (2010; see also Lilly et al. 2013): $\dot{M}_{\text {gas, in }} \simeq 90 \epsilon_{\text {in }}$ $f_{\mathrm{b}, 0.18}\left(M_{\mathrm{h}, 12}\right)^{1.1}((1+z) / 3.2)^{2.2} M_{\odot} \mathrm{yr}^{-1}$. Here $\epsilon_{\mathrm{in}}$ is the accretion efficiency, $f_{\mathrm{b}, 0.18}\left(\equiv f_{\mathrm{b}} / 0.18\right)$ is the cosmic baryonic fraction, and $M_{\mathrm{h}, 12}\left(\equiv M_{\mathrm{h}} / 10^{12} M_{\odot}\right)$ is the mass of the halo. Assuming a fiducial value for the accretion efficiency of $\epsilon_{\text {in }}=0.7$ (e.g., Bauermeister et al. 2010), a cosmic baryonic fraction of $f_{\mathrm{b}}=0.18$, and a halo mass of $M_{\mathrm{h}} \approx 10^{12.5} M_{\odot}$ (based on the relation between halo and stellar mass in Moster et al. 2013), we obtain a mean gas accretion rate for $\mathrm{zC} 400528$ of $\dot{M}_{\mathrm{gas}}$,in $\approx 240 M_{\odot} \mathrm{yr}^{-1}$.

28 The prescriptions for momentum and radiation feedback from AGNs used in Brennan et al. (2018) are presented in Choi et al. (2012, 2015).
}

into the simulated galaxies is suppressed by $\gtrsim 1 \mathrm{dex}$. In addition, the timescale for reaccretion of gas ejected by the outflow increases from $\sim 1$ to $\sim 2-3$ Gyr. If the baryon cycle in $\mathrm{zC} 400528$ is affected by the AGN feedback in a similar way to that described in Brennan et al. (2018), then the gas depletionto-replenishment timescale ratio in $\mathrm{zC} 400528$ should reach the critical level of $t_{\text {rep }} / t_{\text {dep }} \sim 10$, where long-term quenching of the star formation activity is expected.

We can further explore the quenching scenario with a simple back-of-the-envelope calculation that yields a rough estimate of the rate at which a galaxy is moving up or down the MS based on its balance term, which is defined as $B=$ inflow rate/ (SFR + outflow rate). Keeping in mind the many uncertainties associated with the inflow, outflow, and star formation rate measurements, the balance of gas input and drainage in $\mathrm{zC} 400528$ is

$$
B=\frac{240 M_{\odot} \mathrm{yr}^{-1}}{(352+256) M_{\odot} \mathrm{yr}^{-1}} \approx 0.4 .
$$

For simplicity, for the SFR, we have used the average between the $\mathrm{SFR}_{\mathrm{UV} \text {, corr }}$ and $\mathrm{SFR}_{\mathrm{UV}+\mathrm{IR}}$ values. From the scaling relation described in Tacchella et al. (2016) between the balance term $B$ (in the central $5 \mathrm{kpc}$ ) and the rate of change of distance from the MS, $\dot{\Delta}_{\text {MS }}$, we speculate that zC400528 is moving down from the MS at a rate of $\dot{\Delta}_{\mathrm{MS}} \sim 2$ dex Gyr ${ }^{-1}$ (note that the scaling relations in Tacchella et al. 2016 take into account the evolution of the MS of star-forming galaxies with redshift and the reaccretion of gas ejected by outflow episodes). At this rate, it should only take zC400538 1 Gyr to transition from the MS to the regime of passive quenched galaxies $\sim 2$ dex below the MS. This is consistent with the expectations from inside-out quenching that claim that massive star-forming galaxies such as zC400528 should be fully quenched by $z \sim 1$ ( $\sim 3$ Gyr later than the $z \sim 2$ epoch).

\section{Conclusions}

In this paper, we use ALMA $\mathrm{CO}(3-2)$ observations to study the molecular gas properties of the disk and the AGN-driven outflow in $\mathrm{zC} 400528$, a typical massive galaxy at $z=2.3$ in the process of quenching (Tacchella et al. 2015b). We complement these observations with VLT/SINFONI adaptive optics $\mathrm{H} \alpha$ data (Förster Schreiber et al. 2014, 2018a), which allowed us to conduct one of the first spatially resolved, multiphase studies of a galactic outflow at $z \sim 2$.

We highlight the following points.

1. Molecular disk. The molecular gas mass in the rotating disk of zC400528 (assuming a CO-to- $\mathrm{H}_{2}$ conversion factor of $\left.\alpha_{\mathrm{CO}(1-0), \mathrm{T} 18}=4.3 M_{\odot}\left(\mathrm{K} \mathrm{km} \mathrm{s}^{-1} \mathrm{pc}^{-2}\right)^{-1}\right)$ is $M_{\text {mol,disk }}=1.1 \times 10^{11} M_{\odot}$, which translates to a molecular gas-to-stellar ratio of $M_{\mathrm{mol}} / M_{\star} \approx 1$. This high gasmass fraction is comparable to those observed in massive star-forming galaxies at $z \sim 2$ (Genzel et al. 2015; Tacconi et al. 2018).

2. Molecular outflow. The molecular outflow in zC400528 is asymmetrical, with only its receding component detected out to a projected distance of $\sim 10 \mathrm{kpc}$. The bulk of the emission is concentrated in the nuclear region and aligned with the major kinematic axis of the disk. We observe a positive velocity gradient in the outflow with projected velocities that range from $\sim 300 \mathrm{~km} \mathrm{~s}^{-1}$ in the 
nuclear part to $\sim 400-500 \mathrm{~km} \mathrm{~s}^{-1}$ in the furthest components.

We measure a molecular outflow mass and massloss rate of $M_{\text {out,mol }}=3.36 \times 10^{9} M_{\odot}$ and $\dot{M}_{\text {out,mol }}^{\text {avg }} \approx$ $256 M_{\odot} \mathrm{yr}^{-1}$, respectively (these assume a ULIRG-like CO-to- $\mathrm{H}_{2}$ conversion factor). With this powerful molecular mass-loss rate, zC400528 is depleting its nuclear molecular gas at a rate comparable to the star formation activity.

Most likely, the molecular outflow in zC400528 is powered by a combination of star formation and AGN activity, although the latter by itself is sufficient to drive an energy-conserving large-scale outflow ( $\left.\dot{P}_{\text {out,mol }}\right)$ $\left.\left(L_{\mathrm{AGN}, \mathrm{bol}} / c\right) \approx 7, \dot{E}_{\mathrm{out}, \mathrm{mol}} \approx 0.5 \% L_{\mathrm{AGN}, \mathrm{bol}}\right)$.

3. Molecular and ionized phases of the outflow. The ionized and molecular phases of the outflow in $\mathrm{zC} 400528$ have different morphological properties. While the ionized outflow extends for $\sim 4 \mathrm{kpc}$ from the nuclear region inclined about $60^{\circ}$ with respect to the major kinematic axis of the disk, the central component of the molecular outflow is found preferentially in between the ionized wind and the major kinematic axis. This type of configuration is not uncommon in AGN-driven outflows, as found in observational and simulation studies (e.g., García-Burillo et al. 2014; Zubovas \& King 2014). We also observe that in the extended molecular component of the outflow ( $\gtrsim 5 \mathrm{kpc}$ ), there are at least two regions (A and B in Figure 11) where ionized and molecular gas are cospatial and moving at similar high velocities.

Keeping in mind all the uncertainties that affect the determination of the ionized and molecular outflow properties (e.g., electron density, CO-to- $\mathrm{H}_{2}$ conversion factor, geometry, etc.), both the mass and energetics of the outflow are dominated by the molecular phase. For the ionized phase to be dominant would require the combination of low electron densities and optically thin molecular gas in the wind. Both of these conditions seem unlikely (e.g., Aalto et al. 2015; Walter et al. 2017; Förster Schreiber et al. 2018b).

4. AGN-driven outflow quenching in action? Kiloparsecscale observations of the stellar content and star formation activity in $\mathrm{zC} 400528$ reveal that one or more quenching mechanisms are shutting down the star formation activity from the inside out on short timescales ( $\lesssim 1$ Gyr; Tacchella et al. 2015a). The fact that we detect an AGN-driven outflow powerful enough to expel the star-forming material in the central region on short timescales $\left(t_{\text {dep,out }} \sim 0.2 \mathrm{Gyr}\right.$ if we consider the molecular gas in the inner $4 \mathrm{kpc}$ ) points to the outflow as one of the main quenching engines at work. Although it is true that the expelled molecular gas is not fast enough to escape the system, AGN feedback should significantly reduce the accretion of both fresh and recycled gas into the disk (e.g., Brennan et al. 2018), facilitating even more the transition of $\mathrm{zC} 400528$ into the realm of passive systems below the MS of galaxies.

We thank the anonymous referee for very useful comments and suggestions that improved the paper. R.H.C. is grateful for the support and encouragement from Fares Bravo Garrido and dedicates this paper with love to Fares and Olivia. R.H.C. would also like to thank Sandro Tacchella for helpful discussions. S.G.B. is grateful for economic support from funding grant AYA2016-76682-C3-2-P. C.F. acknowledges support from the European Union Horizon 2020 research and innovation program under Marie Sklodowska-Curie grant agreement No. 664931. L.C. acknowledges financial support by the Spanish Ministry for Science, Innovation and Universities under grants AYA2015-68964 and ESP2017-83197. The Cosmic Dawn Center is funded by the Danish National Research Foundation. Part of this work was done in the Parent's Office at the Max Planck Institute for Extraterrestrial Physics, which exists thanks to the initiative of Agata Karska and Heike Modest and the funding from the L'Oréal for Women in Science Prize. This paper makes use of the following ALMA data: ADS/JAO.ALMA\#2013.1.00092.S and \#2015.1.00220.S. ALMA is a partnership of the ESO (representing its member states), NSF (USA), and NINS (Japan), together with the NRC (Canada), NSC and ASIAA (Taiwan), and KASI (Republic of Korea), in cooperation with the Republic of Chile. The Joint ALMA Observatory is operated by the ESO, AUI/NRAO, and NAOJ. This work is based on observations taken by the 3D HST Treasury Program (GO 12177 and 12328) with the NASA/ESA HST, which is operated by the Association of Universities for Research in Astronomy, Inc., under NASA contract NAS5-26555.

\section{Appendix \\ AGN Bolometric Luminosity of zC400528}

Given that $\mathrm{zC} 400528$ is undetected in the Chandra X-ray data of the COSMOS field (Elvis et al. 2009), here we estimate its AGN bolometric luminosity based on the well-known relation between the AGN luminosities in the $2-10 \mathrm{keV}$ energy range and at rest frame $6 \mu \mathrm{m}$ associated with the dusty torus. This relationship is practically linear and has an intrinsic scatter of $\sim 0.35$ dex (Lutz et al. 2004; Mateos et al. 2015). The restframe $7 \mu \mathrm{m}$ luminosity of zC400528, measured from Spitzer MIPS $24 \mu \mathrm{m}$ data (Mancini et al. 2011), is $L_{7 \mu \mathrm{m} \text {,rest }}=1.3 \times$ $10^{45} \mathrm{erg} \mathrm{s}^{-1}$ (Mancini et al. 2011). What we need, however, is the fraction of the $7 \mu \mathrm{m}$ dust luminosity associated with the AGN $\left(L_{7 \mu \mathrm{m}}^{\mathrm{AGN}}\right)$. In a study of 232 AGNs in the redshift range $0.05<z<2.8$, Mateos et al. (2015) found that dust emission at rest frame $6 \mu \mathrm{m}$ is dominated by the AGNs, and in a study of 703 X-ray-selected AGNs in the range $0.1<z<5$, Brown et al. (2018) found that the median AGN contribution to the rest-frame $6 \mu \mathrm{m}$ luminosity is $f_{\mathrm{AGN}} \approx 60 \%$. If we assume the latter, and we use the scaling relation between $L_{7 \mu \mathrm{m}}^{\mathrm{AGN}}$ and $L_{2-10 \mathrm{keV}}^{\mathrm{X} \text { ray }}$ from Mateos et al. (2015), we estimate an X-ray luminosity for zC400528 of $L_{2-10 \mathrm{keV}}^{\mathrm{X}}=3.9 \times 10^{44} \mathrm{erg} \mathrm{s}^{-1}$. Finally, using an $\mathrm{X}$-ray bolometric correction factor of $\kappa_{\text {bol }}\left(\equiv L_{\text {bol }} / L_{\mathrm{X}, 2-10 \mathrm{kev})}\right) 10$ (e.g., Lusso et al. 2012; Brightman et al. 2017), the bolometric AGN luminosity of zC400528 is $L_{\mathrm{AGN} \text {,bol }} \approx 4 \times 10^{45} \mathrm{erg} \mathrm{s}^{-1}$. This value is consistent with the upper limit of $L_{\mathrm{AGN}, \text { bol }} \lesssim 10^{45} \mathrm{erg} \mathrm{s}^{-1}$ from the Chandra X-ray data (calculated assuming $\Gamma=1.9$ and $N_{\mathrm{H}}=10^{22}$ $\mathrm{cm}^{-2}$ ), especially if we consider that this upper limit could be substantially higher in the Compton-thick case. Finally, the $L_{\mathrm{AGN} \text {,bol }}$ is a factor of $\sim 3$ lower than that obtained based on the [N II] $\lambda 6584$ luminosity in the narrow component after subtracting the contribution by star-forming regions based on the mass-metallicity relation (Förster Schreiber et al. 2018b). 


\section{ORCID iDs}

R. Herrera-Camus (1) https://orcid.org/0000-0002-2775-0595

L. Tacconi (i) https://orcid.org/0000-0002-1485-9401

R. Genzel (i) https://orcid.org/0000-0002-2767-9653

N. Förster Schreiber (1) https://orcid.org/0000-0003-

4264-3381

D. Lutz (1) https://orcid.org/0000-0003-0291-9582

A. Bolatto (1) https://orcid.org/0000-0002-5480-5686

S. Wuyts (1) https://orcid.org/0000-0003-3735-1931

A. Renzini (i) https://orcid.org/0000-0002-7093-7355

S. Lilly (1) https://orcid.org/0000-0002-6423-3597

S. Belli (1) https://orcid.org/0000-0002-5615-6018

H. Übler (i) https://orcid.org/0000-0003-4891-0794

T. Shimizu (1) https://orcid.org/0000-0002-2125-4670

E. Sturm (1) https://orcid.org/0000-0002-0018-3666

F. Combes (1) https://orcid.org/0000-0003-2658-7893

S. García-Burillo (1) https://orcid.org/0000-0003-0444-6897

A. Burkert (1) https://orcid.org/0000-0001-6879-9822

T. Naab (1) https://orcid.org/0000-0002-7314-2558

A. Saintonge (1) https://orcid.org/0000-0003-4357-3450

M. Cooper (1) https://orcid.org/0000-0003-1371-6019

A. Weiss 주 https://orcid.org/0000-0003-4678-3939

\section{References}

Aalto, S., Garcia-Burillo, S., Muller, S., et al. 2012, A\&A, 537, A44 Aalto, S., Garcia-Burillo, S., Muller, S., et al. 2015, A\&A, 574, A85 Alatalo, K. 2015, ApJL, 801, L17

Anglés-Alcázar, D., Faucher-Giguère, C.-A., Kereš, D., et al. 2017, MNRAS, 470, 4698

Arribas, S., Colina, L., Bellocchi, E., Maiolino, R., \& Villar-Martín, M. 2014, A\&A, 568, A14

Barro, G., Kriek, M., Pérez-González, P. G., et al. 2016, ApJL, 827, L32

Bauermeister, A., Blitz, L., \& Ma, C.-P. 2010, ApJ, 717, 323

Bolatto, A. D., Warren, S. R., Leroy, A. K., et al. 2013a, Natur, 499, 450

Bolatto, A. D., Warren, S. R., Leroy, A. K., et al. 2015, ApJ, 809, 175

Bolatto, A. D., Wolfire, M., \& Leroy, A. K. 2013b, ARA\&A, 51, 207

Bouché, N., Dekel, A., Genzel, R., et al. 2010, ApJ, 718, 1001

Brennan, R., Choi, E., Somerville, R. S., et al. 2018, ApJ, 860, 14

Brightman, M., Baloković, M., Ballantyne, D. R., et al. 2017, ApJ, 844, 10

Brown, A., Nayyeri, H., Cooray, A., et al. 2018, arXiv:1801.02233

Brusa, M., Cresci, G., Daddi, E., et al. 2018, A\&A, 612, A29

Cecil, G., Dopita, M. A., Groves, B., et al. 2002, ApJ, 568, 627

Choi, E., Ostriker, J. P., Naab, T., \& Johansson, P. H. 2012, ApJ, 754, 125

Choi, E., Ostriker, J. P., Naab, T., Oser, L., \& Moster, B. P. 2015, MNRAS, 449, 4105

Christensen, C. R., Davé, R., Governato, F., et al. 2016, ApJ, 824, 57

Cicone, C., Feruglio, C., Maiolino, R., et al. 2012, A\&A, 543, A99

Cicone, C., Maiolino, R., Sturm, E., et al. 2014, A\&A, 562, A21

Concas, A., Popesso, P., Brusa, M., et al. 2017, A\&A, 606, A36

Contursi, A., Poglitsch, A., Grácia Carpio, J., et al. 2013, A\&A, 549, A118

Cresci, G., Mainieri, V., Brusa, M., et al. 2015, ApJ, 799, 82

Daddi, E., Dannerbauer, H., Liu, D., et al. 2015, A\&A, 577, A46

Dannerbauer, H., Daddi, E., Riechers, D. A., et al. 2009, ApJL, 698, L178

Dasyra, K. M., Combes, F., Oosterloo, T., et al. 2016, A\&A, 595, L7

Di Matteo, T., Springel, V., \& Hernquist, L. 2005, Natur, 433, 604

Eisenhauer, F., Abuter, R., Bickert, K., et al. 2003, Proc. SPIE, 4841, 1548

Elvis, M., Civano, F., Vignali, C., et al. 2009, ApJS, 184, 158

Fan, L., Knudsen, K. K., Fogasy, J., \& Drouart, G. 2018, ApJL, 856, L5

Faucher-Giguère, C.-A., \& Quataert, E. 2012, MNRAS, 425, 605

Feruglio, C., Fiore, F., Carniani, S., et al. 2015, A\&A, 583, A99

Feruglio, C., Maiolino, R., Piconcelli, E., et al. 2010, A\&A, 518, L155

Fiore, F., Feruglio, C., Shankar, F., et al. 2017, A\&A, 601, A143

Fluetsch, A., Maiolino, R., Carniani, S., et al. 2019, MNRAS, 483, 4586

Förster Schreiber, N. M., Genzel, R., Newman, S. F., et al. 2014, ApJ, 787, 38

Förster Schreiber, N. M., Renzini, A., Mancini, C., et al. 2018a, ApJS, 238, 21

Förster Schreiber, N. M., Übler, H., Davies, R. L., et al. 2018b, arXiv:1807. 04738

Gabor, J. M., \& Bournaud, F. 2014, MNRAS, 441, 1615
Gabor, J. M., Davé, R., Oppenheimer, B. D., \& Finlator, K. 2011, MNRAS, 417, 2676

García-Burillo, S., Combes, F., Usero, A., et al. 2014, A\&A, 567, A125

Genzel, R., Förster Schreiber, N. M., Rosario, D., et al. 2014, ApJ, 796, 7

Genzel, R., Tacconi, L. J., Lutz, D., et al. 2015, ApJ, 800, 20

González-Alfonso, E., Fischer, J., Graciá-Carpio, J., et al. 2014, A\&A, 561, A27

González-Alfonso, E., Fischer, J., Spoon, H. W. W., et al. 2017, ApJ, 836, 11

Harrison, C. M., Alexander, D. M., Mullaney, J. R., et al. 2016, MNRAS, 456, 1195

Heckman, T. M., Alexandroff, R. M., Borthakur, S., Overzier, R., \& Leitherer, C. 2015, ApJ, 809, 147

Herrera-Camus, R., Bolatto, A. D., Wolfire, M. G., et al. 2015, ApJ, 800, 1

Hibbard, J. E., van der Hulst, J. M., Barnes, J. E., \& Rich, R. M. 2001, AJ, 122,2969

Janssen, A. W., Christopher, N., Sturm, E., et al. 2016, ApJ, 822, 43

Kennicutt, R. C., \& Evans, N. J. 2012, ARA\&A, 50, 531

Kewley, L. J., Maier, C., Yabe, K., et al. 2013, ApJL, 774, L10

King, A., \& Pounds, K. 2015, ARA\&A, 53, 115

King, A. R. 2010, MNRAS, 402, 1516

Kirkpatrick, A., Pope, A., Charmandaris, V., et al. 2013, ApJ, 763, 123

Kornei, K. A., Shapley, A. E., Martin, C. L., et al. 2012, ApJ, 758, 135

Lacy, M., Storrie-Lombardi, L. J., Sajina, A., et al. 2004, ApJS, 154, 166

Leroy, A. K., Bigiel, F., de Blok, W. J. G., et al. 2012, AJ, 144, 3

Leroy, A. K., Walter, F., Martini, P., et al. 2015, ApJ, 814, 83

Leung, G. C. K., Coil, A. L., Azadi, M., et al. 2017, ApJ, 849, 48

Lilly, S. J., Carollo, C. M., Pipino, A., Renzini, A., \& Peng, Y. 2013, ApJ, 772,119

Lusso, E., Comastri, A., Simmons, B. D., et al. 2012, MNRAS, 425, 623

Lutz, D., Maiolino, R., Spoon, H. W. W., \& Moorwood, A. F. M. 2004, A\&A, 418,465

Maiolino, R., Gallerani, S., Neri, R., et al. 2012, MNRAS, 425, L66

Mancini, C., Förster Schreiber, N. M., Renzini, A., et al. 2011, ApJ, 743, 86

Mateos, S., Carrera, F. J., Alonso-Herrero, A., et al. 2015, MNRAS, 449, 1422

McMullin, J. P., Waters, B., Schiebel, D., Young, W., \& Golap, K. 2007, in ASP Conf. Ser. 376, Astronomical Data Analysis Software and Systems XVI, ed. R. A. Shaw, F. Hill, \& D. J. Bell (San Francisco, CA: ASP), 127

Morganti, R., Veilleux, S., Oosterloo, T., Teng, S. H., \& Rupke, D. 2016, A\&A, 593, A30

Moster, B. P., Naab, T., \& White, S. D. M. 2013, MNRAS, 428, 3121

Muratov, A. L., Kereš, D., Faucher-Giguère, C.-A., et al. 2015, MNRAS, 454, 2691

Murphy, E. J., Condon, J. J., Schinnerer, E., et al. 2011, ApJ, 737, 67

Murray, N., Quataert, E., \& Thompson, T. A. 2005, ApJ, 618, 569

Muzzin, A., Marchesini, D., Stefanon, M., et al. 2013, ApJ, 777, 18

Narayanan, D., Turk, M., Feldmann, R., et al. 2015, Natur, 525, 496

Nardini, E., Reeves, J. N., Gofford, J., et al. 2015, Sci, 347, 860

Nelson, E. J., van Dokkum, P. G., Förster Schreiber, N. M., et al. 2016, ApJ, 828,27

Newman, S. F., Buschkamp, P., Genzel, R., et al. 2014, ApJ, 781, 21

Newman, S. F., Genzel, R., Förster Schreiber, N. M., et al. 2013, ApJ, 767, 104 Oppenheimer, B. D., Davé, R., Kereš, D., et al. 2010, MNRAS, 406, 2325

Pereira-Santaella, M., Colina, L., García-Burillo, S., et al. 2016, A\&A, 594, A81

Pereira-Santaella, M., Colina, L., Garcia-Burillo, S., et al. 2018, A\&A, 6161, A171

Pettini, M., \& Pagel, B. E. J. 2004, MNRAS, 348, L59

Pilbratt, G. L., Riedinger, J. R., Passvogel, T., et al. 2010, A\&A, 518, L1

Poglitsch, A., Waelkens, C., Geis, N., et al. 2010, A\&A, 518, L2+

Richings, A. J., \& Faucher-Giguère, C.-A. 2018, MNRAS, 474, 3673

Rodríguez Zaurín, J., Tadhunter, C. N., Rose, M., \& Holt, J. 2013, MNRAS, 432, 138

Rupke, D. S., Veilleux, S., \& Sanders, D. B. 2002, ApJ, 570, 588

Rupke, D. S., Veilleux, S., \& Sanders, D. B. 2005, ApJ, 632, 751

Rupke, D. S. N., \& Veilleux, S. 2011, ApJL, 729, L27

Schawinski, K., Koss, M., Berney, S., \& Sartori, L. F. 2015, MNRAS, 451,2517

Schinnerer, E., Sargent, M. T., Bondi, M., et al. 2010, ApJS, 188, 384

Silk, J., \& Rees, M. J. 1998, A\&A, 331, L1

Skelton, R. E., Whitaker, K. E., Momcheva, I. G., et al. 2014, ApJS, 214, 24

Smith, K. L., Mushotzky, R. F., Vogel, S., et al. 2016, ApJ, 832, 163

Springel, V., Di Matteo, T., \& Hernquist, L. 2005, ApJL, 620, L79

Sturm, E., González-Alfonso, E., Veilleux, S., et al. 2011, ApJL, 733, L16

Tacchella, S., Carollo, C. M., Förster Schreiber, N. M., et al. 2018, ApJ, 859,56

Tacchella, S., Carollo, C. M., Renzini, A., et al. 2015a, Sci, 348, 314 
Tacchella, S., Dekel, A., Carollo, C. M., et al. 2016, MNRAS, 457, 2790

Tacchella, S., Lang, P., Carollo, C. M., et al. 2015b, ApJ, 802, 101

Tacconi, L. J., Genzel, R., Neri, R., et al. 2010, Natur, 463, 781

Tacconi, L. J., Genzel, R., Saintonge, A., et al. 2018, ApJ, 853, 179

Tacconi, L. J., Neri, R., Genzel, R., et al. 2013, ApJ, 768, 74

Tecza, M., Thatte, N., \& Maiolino, R. 2001, in IAU Symp. 205, Galaxies and their Constituents at the Highest Angular Resolutions, ed. R. T. Schilizzi (Cambridge: Cambridge Univ. Press), 216

Tombesi, F., Meléndez, M., Veilleux, S., et al. 2015, Natur, 519, 436

Übler, H., Naab, T., Oser, L., et al. 2014, MNRAS, 443, 2092

van de Voort, F., Schaye, J., Booth, C. M., Haas, M. R., \& Dalla Vecchia, C. 2011, MNRAS, 414, 2458
Vayner, A., Wright, S. A., Murray, N., et al. 2017, ApJ, 851, 126

Veilleux, S., Bolatto, A., Tombesi, F., et al. 2017, ApJ, 843, 18

Veilleux, S., Cecil, G., \& Bland-Hawthorn, J. 2005, ARA\&A, 43, 769

Walter, F., Bolatto, A. D., Leroy, A. K., et al. 2017, ApJ, 835, 265

Whitaker, K. E., Franx, M., Leja, J., et al. 2014, ApJ, 795, 104

Wong, O. I., Koss, M. J., Schawinski, K., et al. 2016, MNRAS, 460, 1588

Woo, J.-H., Bae, H.-J., Son, D., \& Karouzos, M. 2016, ApJ, 817, 108

Zschaechner, L. K., Bolatto, A. D., Walter, F., et al. 2018, ApJ, 867, 111

Zubovas, K., \& King, A. 2012, ApJL, 745, L34

Zubovas, K., \& King, A. 2016, MNRAS, 462, 4055

Zubovas, K., \& King, A. R. 2014, MNRAS, 439, 400

Zubovas, K., \& Nayakshin, S. 2014, MNRAS, 440, 2625 